\title{
Cassini UVIS Solar occultations by Saturn's F ring and the detection of collision-produced micron-sized dust
}

\author{
Tracy M. Becker ${ }^{\mathrm{a}, \mathrm{b}}$, Joshua E. Colwell ${ }^{\mathrm{b}}$, Larry W. Esposito ${ }^{\mathrm{c}}$, Nicholas O. \\ Attree $^{\mathrm{d}, \mathrm{e}}$, Carl D. Murray ${ }^{\mathrm{e}}$ \\ ${ }^{a}$ Southwest Research Institute, 6220 Culebra Rd. San Antonio, TX, 78238, USA \\ ${ }^{b}$ Department of Physics and Florida Space Institute, University of Central Florida, 4111 \\ Libra Drive, Physical Sciences Bldg. 430, Orlando, FL 32816-2385, USA \\ ${ }^{c}$ LASP, University of Colorado, 392 UCB Boulder, CO, 80309-0392, USA \\ ${ }^{d}$ Aix Marseille Univ., CNRS, LAM, Laboratoire d'Astrophysique de Marseille, Marseille, \\ France \\ ${ }^{e}$ Queen Mary University of London, Mile End Road, London E1 4NS, UK
}

\begin{abstract}
We present an analysis of eleven solar occultations by Saturn's F ring observed by the Ultraviolet Imaging Spectrograph (UVIS) on the Cassini spacecraft. In four of the solar occultations we detect an unambiguous signal from diffracted sunlight that adds to the direct solar signal just before or after the occultations occur. The strongest detection was a $10 \%$ increase over the direct signal that was enabled by the accidental misalignment of the instrument's pointing. We compare the UVIS data with images of the F ring obtained by the Cassini Imaging Science Subsystem (ISS) and find that in each instance of an unambiguous diffraction signature in the UVIS data, the ISS data shows that there was a recent disturbance in that region of the F ring. Similarly, the ISS images show a quiescent region of the F ring for all solar occultations in which no diffraction signature was detected. We therefore conclude that collisions in the $\mathrm{F}$ ring produce a population of small ring particles that can produce a detectable diffraction signal immediately interior or exterior to the $\mathrm{F}$ ring. The clearest example of this connection comes from the strong detection of diffracted light in the 2007 solar occultation, when the portion of the F ring that occulted the Sun had suffered a large collisional event with S/2004 S 6 several months prior. This collision was observed in a series of ISS images (Murray, C.D. et al. [2008]. Nature 453, 739-744).
\end{abstract}

Our spectral analysis of the data shows no significant spectral features 
in the $\mathrm{F}$ ring, indicating that the particles must be at least 0.2 microns in radius. We apply a forward model of the solar occultations, accounting for the effects of diffracted light and the attenuated direct solar signal, to model the observed solar occultation light curves. These models constrain the optical depth, radial width, and particle size distribution of the $\mathrm{F}$ ring. We find that when the diffraction signature is present, we can best reproduce the occultation data using a particle population with an average effective particle size of less than 300 microns, while occultations without clear diffraction signals are best modeled using a population with an effective particle size larger than 400 microns.

Keywords: Planetary rings, occultations, Saturn

\section{Introduction}

Saturn's F ring lies approximately $3,000 \mathrm{~km}$ beyond the main ring system, with a semi-major axis of $\sim 140,223 \mathrm{~km}$ (Bosh et al., 2002; Albers et al., 2012). This multifaceted ring is comprised of a low-optical depth, 10 - 50 $\mathrm{km}$-wide core and a discontinuous, optically thick, 1-km wide "true core" (Murray et al., 2008; Albers et al., 2012), all embedded within an opticallythin envelope of sub-millimeter-sized dust extending $580 \pm 70 \mathrm{~km}$ radially (Showalter et al., 1992; French et al., 2012). Additionally, a core-crossing spiral structure is often observed as faint strands that vary in shape, optical depth, and number, on either or both sides of the F ring core (Charnoz et al., 2005; Murray et al., 2005; Charnoz et al., 2009; Albers et al., 2012).

The $\mathrm{F}$ ring is primarily composed of dust; analyses of the backscattered signal in Hubble Space Telescope (HST), ground-based observations (Poulet et al., 2000), and high phase-angle Voyager observations (Showalter et al., 1992 ) indicate $80 \%-98 \%$ dust fraction. Vahidinia et al. (2011) report a narrow size distribution of particles with radii between 20-30 micrometers based on data from the Cassini Visual and Infrared Mapping Spectrometer (VIMS). A VIMS spectral analysis by Hedman et al. (2011) revealed a two-component size distribution, where particles between 10 microns and 1 millimeter followed a size distribution typical for a population that has evolved through collisional disruption (Dohnanyi, 1969), and for particles smaller than 10 microns, the slope of the size distribution was much shallower. Ultraviolet stellar occultation data from the Faint Object Spectrometer on the HST placed a lower limit on the minimum particle size in the F ring of approximately 10 
microns (Bosh et al., 2002).

Small particles are expected to sputter away in $10^{4}$ years or be eroded by micrometeoroid impact bombardment in $10^{6}$ years (Grün et al., 1984; Burns et al., 1984; Showalter et al., 1992). The persistence of such small particles is therefore indicative of ongoing activity in the $\mathrm{F}$ ring, namely collisions into large objects in the $\mathrm{F}$ ring core. Evidence for such large, progenitor objects has been mounting; Pioneer 11 indirectly detected a belt of objects $0.1-1$ $\mathrm{km}$ in size spanning a 2000-km-wide region surrounding the ring through a measurement of the depletion in the flux of trapped magnetospheric electrons (Cuzzi and Burns, 1988). In the narrow component of the $\mathrm{F}$ ring core, objects on the order of a kilometer in size have been directly imaged by the Cassini Imaging Science Subsystem (ISS) (Murray et al., 2008). Additionally, detections of ephemeral clumps or moonlets in UV stellar occultation data from the Cassini Ultraviolet Imaging Spectrograph (UVIS) led to the prediction that up to 140,000 objects on the order of 600 meters in size exist in this region (Esposito et al., 2008; Meinke et al., 2012).

Large objects such as S/2004 S6 have been observed on core-crossing orbits (Porco et al., 2005; Spitale et al., 2006), and large physical collisions of such bodies with core-embedded objects are believed to produce the massive jets that form the observed $\mathrm{F}$ ring spiral structure (Charnoz et al., 2005; Murray et al., 2008; Charnoz et al., 2009). Lower-velocity collisions ( 1 $\mathrm{m} / \mathrm{s}$ ) may be responsible for the small, irregular features in the core known as mini-jets that were catalogued by Attree et al. (2014). The particle size distribution in the $\mathrm{F}$ ring is a useful constraint on the frequency and energy of these impacts.

We find evidence for the presence (and varying quantity) of micron-sized dust in UVIS observations of solar occultations by Saturn's F ring. During the first solar occultation measurement of the rings by UVIS in 2005, the boresight of the UVIS solar port was not properly pointed at the Sun. The unocculted solar signal was reduced to $\leq 2.5 \%$ its nominal signal because the majority of the solar disk was outside the instrument's field of view. The diminished overall brightness of the Sun enabled the serendipitous detection of light diffracted by the particles in Saturn's F ring. The signal due to diffraction was $10 \%$ brighter than the unocculted signal. We also find unambiguous diffraction signal in four properly pointed solar occultations (Sun centered in the field of view). The shape and intensity of the diffraction signatures are controlled by the size distribution of the particles that are diffracting the light. 
In this paper, we constrain the particle size distribution, optical depth, and radial width of the F ring through (1) the UV spectral analysis of the ring from Cassini UVIS solar occultation data and (2) computational models of the light diffracted by small ring particles during UVIS solar occultations. We begin by describing the UVIS occultation data in Section 2. In Section 3 we discuss the collisional features in the ISS images captured just before or after the solar occultations and compare with the UVIS detections of unambiguous diffraction signatures. Section 4 describes the theoretical background for the diffraction of light that we apply to our computational model. In Section 5 we present a spectral analysis of the F ring occultation data. We describe our diffraction model of the F ring in Section 6. Finally, we present our results in Section 7 and summarize our conclusions in Section 8.

\section{UVIS Observations}

As of 2016, UVIS observed 11 solar occultations by Saturn's F ring. Information about each solar occultation is listed in Table 1. Throughout this work, we label each observation according to the revolution (Rev) number of Cassini around Saturn and whether the occultation was ingress (I) or egress (E), where ingress occultations are those in which the intercept point of the sunlight in the ring plane approaches Saturn, and egress occultations are when the point recedes from the planet. Solar occultation experiments are conducted by pointing the solar port of the UVIS instrument toward the Sun as the rings pass between the Sun and the spacecraft. Sunlight enters through a small aperture $20^{\circ}$ from the instrument's nominal boresight and is redirected and dispersed onto the detector by a cylindrical grazing incidence mirror (Esposito et al., 2004). Solar occultations are observed with the UVIS extreme ultraviolet (EUV) channel. The EUV spectrograph has a bandpass of 56 - $118 \mathrm{~nm}$ with 1024 spectral elements.

Due to the dispersing mirror, there is no spatial resolution during a solar occultation along the detector's 64 spatial elements. To obtain the highest signal to noise ratio we sum over the spectral elements. We discuss an independent spectral analysis of the data in Section 5. We present all of the occultations as a function of distance from the center of Saturn (ring plane radius) in Figure 1. The solar occultations were observed with integration periods from 1.0 - 5.5 seconds. We have binned the occultations with 1-second integration periods into 4-second bins and normalized all the data sets to instrument counts per second. The central feature in each observation is a 
result of the sun being occulted by the F ring. In the Rev 59 (I), Rev 65 (I), and Rev 66 (I) plots, when the line of sight distances are greatest, the low points interior to the $\mathrm{F}$ ring are due to the beginning of the solar occultation by the A ring.

Some of the data show trends in the unocculted solar signal, possibly due to a slight drift of the Sun in the instrument's field of view (FOV). We fit a line to the unocculted solar signal beyond the F ring $(\geq 145,500 \mathrm{~km}$ - 170,000 km) to determine the baseline signal for each observation (horizontal gray line in each plot). We then remove the trend from the data and determine which, if any, data points are a $2 \sigma$ (magenta plus signs) or $3 \sigma$ (blue diamonds) increase in signal over the baseline (assuming a Poisson distribution where $1 \sigma=\sqrt{N}$, where $N$ is the mean of the baseline signal), indicative of diffraction by the $\mathrm{F}$ ring particles.

The first plot in Figure 1 shows the data from the misaligned Rev 9 occultation that resulted in a 10\% increase over the unocculted signal near the $\mathrm{F}$ ring core. Note that because of the instrument misalignment, the FOV was placed almost entirely off the disk of the Sun, reducing the solar signal to 2,000 counts per second compared with $\sim 80,000$ counts per second in the other occultations. There seems to be some indication that interior to the F ring, the signal does not completely return to the unocculted signal level. It is not clear what causes this, but it could be due to a slight drift in the Sun's position in the FOV or it could be associated with diffraction from the A ring.

Some of the properly pointed solar occultations also show a signal above the baseline signal in the vicinity of the $\mathrm{F}$ ring due to diffracted light. We interpret all solar occultations with at least one $3 \sigma$ detection of signal above the unocculted baseline solar signal to be possible detections of UVIS diffraction signatures. We also interpret occultations with more than one $2 \sigma$ detections within 2,500 $\mathrm{km}$ of the $\mathrm{F}$ ring core to be possible detections of UVIS diffraction signatures. Thus, all occultations with the exception of Rev 59 (I) and Rev 62 (I) are considered possible detections (Figure 1). Further, if multiple high-confidence detections are observed within the $2,500 \mathrm{~km}$ of the $\mathrm{F}$ ring core, with at least one of them being a $3 \sigma$ detection, then we assert that the occultation shows a positive detection of a diffraction signature. These occultations include Rev 9 (I), Rev 43 (E), Rev 55 (E) and Rev 62 (E). We note that although Rev 66 (I) and Rev 66 (E) each show at least one detection of a $3 \sigma$ detection, this detection is farther from the $\mathrm{F}$ ring core and could potentially be associated with the edge of the A ring. 
In addition to diffraction detections, Figure 1 shows that there is variation in the shape of the occultation light curves. All plots (with the exception of Rev 9 because of the offset) are plotted on the same scale. These variations in depth and width are due to both the geometry of the occultations and the intrinsic variation of the $\mathrm{F}$ ring itself.

\section{ISS Images}

For context, we compare the state of the F ring during each UVIS solar occultations with ISS images of the ring. Figure 2 shows mosaics of the Cassini ISS images re-projected relative to a model of the F ring from Cooper et al. (2013). The mosaics are in co-rotating longitude and were produced using an assumed mean motion of $581.96 \mathrm{deg} /$ day for the $\mathrm{F}$ ring with an epoch of 2007 January 01 12:00:00.0 UTC. The longitude scale in each mosaic covers 0 to 360 degrees and the vertical scale is radial distance from $-750 \mathrm{~km}$ to $+750 \mathrm{~km}$ with respect to the Fit 11 orbit model from Table 3 of Cooper et al. (2013). For all mosaics presented in this work, the horizontal axis is the longitude in a frame co-rotating at the ring's mean motion and the vertical axis is the orbital radius from Saturn relative to the ring orbit model (therefore taking into account its eccentricity).

The mosaics were selected due to their proximity in time to the UVIS solar occultations analyzed in this study. At the top right of each mosaic, we include the solar occultation Rev number and the difference in time between when the ISS images were acquired and when the UVIS occultation occurred. A negative value indicates that the ISS mosaic images were acquired before the occultation occurred, whereas a positive value indicates the number of days after the occultation that the ISS images were acquired. The mosaics also show a yellow vertical line that indicates the inertial longitude of the $\mathrm{F}$ ring that occulted the Sun during the UVIS solar occultation observation. Details about the mosaics are provided in Table 2.

We assess each mosaic for irregular features, such as jets and mini-jets, near the ring longitude of the UVIS occultation for signs of recent collisions within the F ring (Murray et al., 2008; Attree et al., 2014). We find that in five instances the region of the F ring that occulted the Sun was near a recent collisional event. These occultations include Rev 9 (I), Rev 43 (E), Rev 55 (E), and Rev 62 (E). There were possible collisions near the occulting ring 

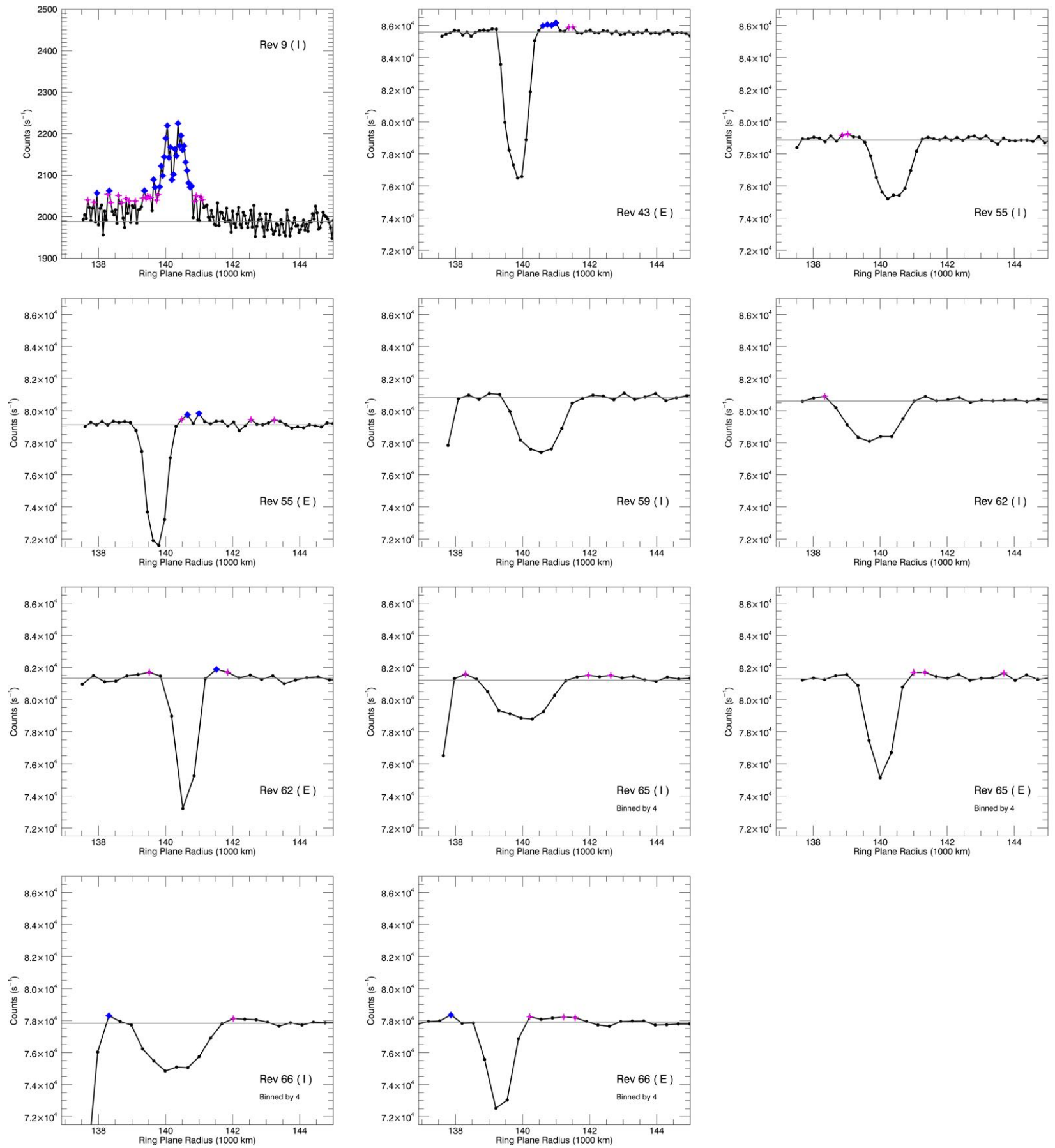

Figure 1: UVIS solar occultations by Saturn's F ring. We bin all occultations with 1second integration periods to 4 seconds for more direct comparison with the other data. Binned data are noted on the plots. All other occultations had a 4-second integration period, with the exception of Rev 43 which had a 5.5-second integration period. The data are normalized to show counts per second as a function of ring plane radius from Saturn. The gray horizontal line is the baseline of the unocculted solar signal. Magenta ' + ' mark data points that are $2 \sigma$ detections and blue diamonds mark $3 \sigma$ detections of an enhanced signal above the unocculted solar signal baseline. Such detections just inside or outside of the $\mathrm{F}$ ring light curve suggest that these signals are the combination of the direct solar signal plus additional sunlight that has been diffracted by small $\mathrm{F}$ ring particles into the UVIS FOV. 
Table 1: UVIS Solar Occultations by Saturn's F ring

\begin{tabular}{lccccc}
\hline Reference & $\begin{array}{c}\text { Integration } \\
(\mathrm{s})\end{array}$ & $\begin{array}{c}B \text { angle } \\
(\text { Degrees })\end{array}$ & $\begin{array}{c}\text { Inertial Longitude } \\
(\text { Degrees })\end{array}$ & $\begin{array}{c}\text { Line-of-Sight Distance } \\
(\mathrm{km})\end{array}$ & $\begin{array}{c}\text { Date } \\
\text { Year-DOY }\end{array}$ \\
\hline Rev 9 (I) & 4.0 & 20.96 & 251.3 & 207,996 & $2005-159$ \\
Rev 43 (E) & 5.5 & 12.66 & 75.1 & 347,275 & $2007-114$ \\
Rev 55 (I) & 4.0 & 8.97 & 260.9 & 352,124 & $2008-003$ \\
Rev 55 (E) & 4.0 & 8.97 & 353.4 & 150,022 & $2008-003$ \\
Rev 59 (I) & 4.0 & 8.24 & 243.4 & 324,410 & $2008-051$ \\
Rev 62 (I) & 4.0 & 7.77 & 245.6 & 350,650 & $2008-083$ \\
Rev 62 (E) & 4.0 & 7.77 & 183.6 & 112,221 & $2008-083$ \\
Rev 65 (I) & 1.0 & 7.34 & 249.5 & 376,039 & $2008-111$ \\
Rev 65 (E) & 1.0 & 7.34 & 355.9 & 150,742 & $2008-111$ \\
Rev 66 (I) & 1.0 & 7.20 & 249.9 & 375,645 & $2008-121$ \\
Rev 66 (E) & 1.0 & 7.20 & 355.3 & 151,450 & $2008-121$ \\
\hline
\end{tabular}

List of Cassini UVIS solar occultations analyzed in this study. Integration is the integration period of the instrument for each occultation. The $B$ angle is the ring opening angle. The inertial longitude is the longitude of the $\mathrm{F}$ ring where the line-of-sight vector of the UVIS boresight intersects the ring, which is measured prograde from the ascending node of Saturn's equatorial plane on Earth's J2000 equator. The line of sight distance is the distance between Cassini and the ring plane at the time of the observation. The date indicates the year and day-of-year (DOY) that the observation occurred. 
region during Rev 65 (E), Rev 66 (E) and a possibly older, evolved collision in the occulting region during Rev 55 (I). There are four mosaics that show no collisional activity near the UVIS occultation longitude: Rev 59 (I), Rev 62 (I), Rev 65 (I), and Rev 66 (I).

We compare the UVIS diffraction signature detections and the ISS collision detections in Table 3. This table shows that for every UVIS solar occultation in which a UVIS diffraction signature was detected, a collisional feature was also observed in the ISS images and for every UVIS occultation during which no diffraction was detected, no collisional features were observed in the corresponding ISS images. For UVIS occultations with ambiguous but possible detections of diffraction, the detection of collisional features in the ISS images vary. This result shows a strong correlation between the presence of diffraction in UVIS occultation data, presumably from the release of smaller ring particles, and a collisional event in Saturn's F ring.

Some of the collision events are particularly notable. For example, we find that during Rev 43, the region of the F ring that occulted the Sun was serendipitously the same region studied extensively by Murray et al. (2008) due to the detection of a large collision or series of collisions. They show a series of images of this ring location as the large collision event evolved from December 2006 through May 2007. They assert that this feature was produced by a collision in the core with a core-crossing, $5-\mathrm{km}$ object dubbed S/2004 S 6, or some fragment of it. French et al. (2012) discuss how this collision event increased the mean brightness of the $\mathrm{F}$ ring by $84 \%$, with the brightening effects decaying thereafter with a half life of 91 days. The UVIS observation of this region occurred in April of 2007 and resulted in the clearest detection of diffracted light of any of the properly-aligned solar occultations, further verifying the connection between UVIS diffraction signatures and collisional events in the $\mathrm{F}$ ring.

Also of note is the Rev 9 solar occultation. The ISS mosaic shows that a large F ring collision event, similar to that seen near the time of the Rev 43 occultation, occurred just upstream of the ring region observed during Rev 9. This may imply that it was not just the offset of the UVIS solar port, but also proximity to a large collisional event, that enabled the detection of diffracted light during the Rev 9 occultation. 

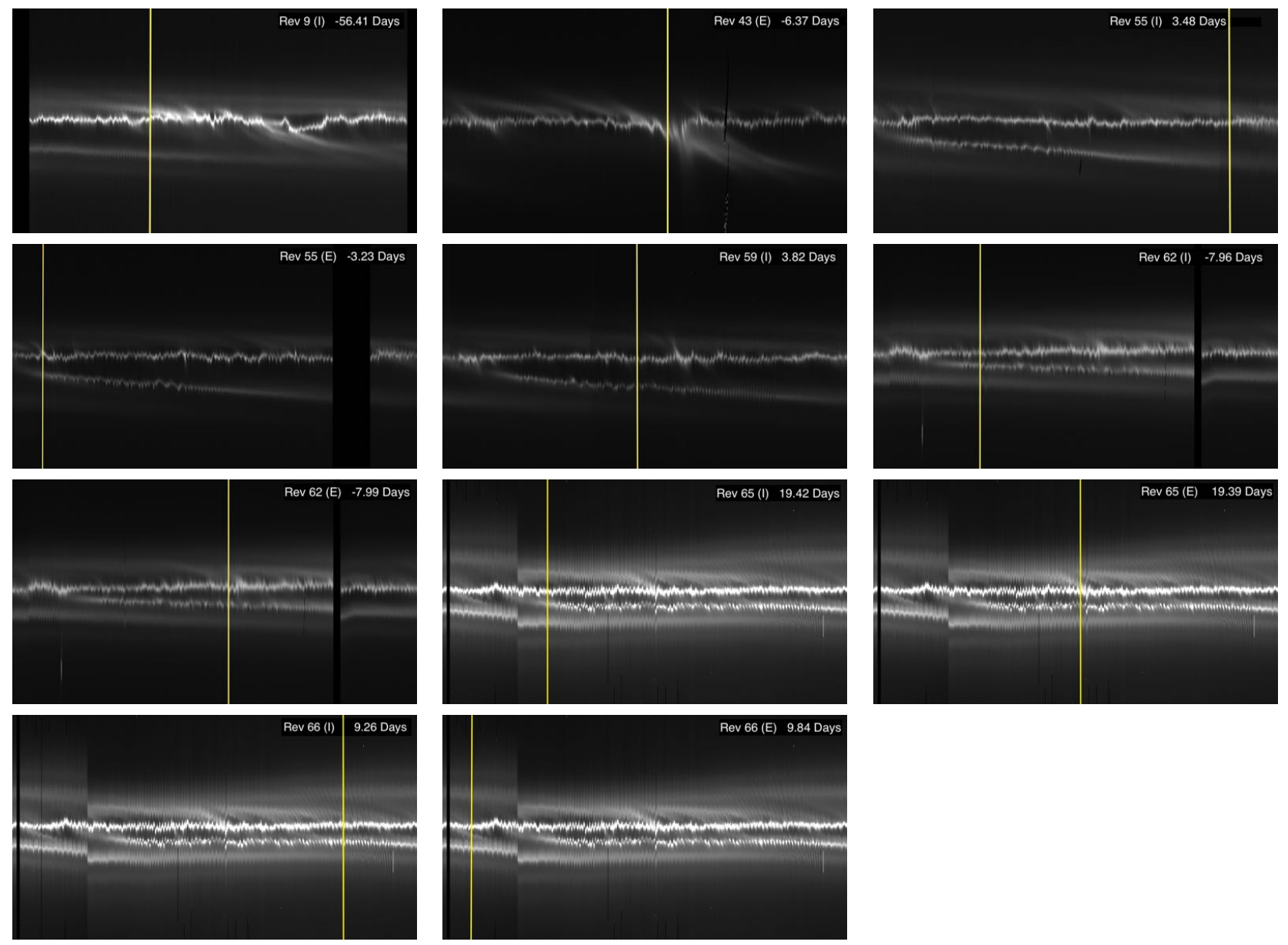

Figure 2: ISS image mosaics of the $\mathrm{F}$ ring at times near when the solar occultations occurred. The horizontal axis is the longitude in a frame corotating at the ring's mean motion and the vertical axis is the orbital radius from Saturn relative to the ring orbit model. At the top right of each mosaic, we include the solar occultation Rev number closest to when the images were acquired and what the difference in time between between the images and solar occultation was. A negative number of days indicates that the ISS images occurred before the occultation, whereas a positive number of days indicates that the images were taken after the occultation. The mosaics also show a yellow vertical line that indicates the inertial longitudinal location of the UVIS solar occultation converted to a co-rotating longitude for plotting purposes. 


\section{Theory}

The light curves observed during the F ring solar occultations (Fig. 1) are the combination of two processes: (1) the attenuation of the direct solar signal and (2) the addition of solar photons from outside the direct solar line-of-sight that are diffracted into the instrument's FOV. The attenuation of the solar signal is caused by the absorption and scattering of UV photons by the ring particles as they occult the Sun. This includes light that is lost due to particles diffracting light out of the FOV (Section 4.2). The addition of indirect sunlight is apparent as signal above the direct solar signal baseline observed in several of the occultation data sets. This additional signal is the result of particles diffracting sunlight into the instrument FOV. Here, the term diffraction is used to indicate Fraunhofer diffraction, which is described in the following section. The intensity of the Fraunhofer diffraction pattern is dependent on the particle size $(a)$ and the wavelength $(\lambda)$ of light. As a result, measurements of an observed diffraction signal (for a given wavelength) can be used to derive the sizes of the particles responsible for the diffraction. This technique has been used to measure the particle size distribution in the rings of Saturn (e.g. French and Nicholson (2000); Harbison et al. (2013); Becker et al. (2016)) and of Uranus (Cuzzi, 1985).

\subsection{Fraunhofer Diffraction}

In this work we assume that the ring particles are well-defined, separate entities, such that the particles are independent scatterers. An agglomerate of small particles can be treated as one, larger particle. We focus on light attenuated and scattered by particles with radii between $1 \mu \mathrm{m}$ and $1 \mathrm{~mm}$ in size and are therefore much larger than the incident wavelength of light $(\sim$ $100 \mathrm{~nm}$ ). Because $\frac{2 \pi a}{\lambda}>>1$, one can relatively easily separate the light into "scattered" and "diffracted" components. This is known as the geometric optics regime. The angular distribution of the intensity of the diffracted light by such particles is described by Fraunhofer diffraction (van de Hulst, 1957).

The Fraunhofer diffraction pattern is produced assuming all particles are spheres, which is somewhat simplistic for the particles in Saturn's rings. However, unless the particles are significantly oblong and also have a preferential orientation (unlikely for the small particles producing the observed diffraction signal), this assumption does not significantly impact our conclusions 
about the particle sizes (van de Hulst, 1957). We also assume the observations occur in the far-field, defined as $D>>\frac{2 a^{2}}{\lambda}$, where $D$ is the distance of Cassini from the ring particles, which is satisfied for all solar occultations distances and particle sizes discussed in this work.

The Fraunhofer diffraction pattern is produced when a plane wave front encounters an object. An object with radius $a$ blocks an amount of light equivalent to its geometric cross section, $G_{\text {scatt }}=\pi a^{2}$. $G_{\text {scatt }}$ refers strictly to the amount of light scattered and absorbed by the object, and does not include diffracted light. Here, the geometric area is the 2-dimensional area of the particles that a plane wave would encounter. Huygens' principle causes the incomplete wave front to produce, at large distances, the Fraunhofer diffraction pattern (van de Hulst, 1957). This diffraction pattern is more commonly associated with the result of light passing through an aperture, like that of a telescope, and is known as the "Airy disk". According to Babinet's principle, if the area of the aperture is the same as the geometric area of the particle, the resulting intensities of the diffraction patterns are identical (van de Hulst, 1957). Therefore spherical particles (like those assumed here for Saturn's rings) will produce this characteristic "Airy disk" diffraction pattern.

To first order, the angular size $\theta$ of the Fraunhofer diffraction pattern is

$$
\theta=\frac{1.22 \lambda}{2 a}
$$

For relatively large particles the diffraction cone subtends a very small angle, while small particles produce diffraction cones with a large angular extent. Because the particles are in the geometric optics regime, the diffraction pattern is dependent exclusively on the particle size and the incident wavelength, and is independent of the particle composition. It also removes the need to understand the albedo and scattering properties of Saturn's rings in the modeling efforts presented in this work.

\subsection{Extinction Efficiency}

Babinet's principle gives rise to the Extinction Paradox, sometimes known as Babinet's Paradox. The Extinction Paradox states that the total energy removed by a particle in the geometric optics regime will have an effective cross section equal to twice the geometric area, $2 G_{\text {scatt }}$, of the particle intercepting the light (van de Hulst, 1957). As the incident light encounters a 
particle, the particle will absorb and/or scatter (through reflection and refraction) an amount of energy with an effective cross section equal to $G_{\text {scatt }}$. Additionally, the particle will also diffract light in a way that mimics diffraction through an aperture with area $G_{\text {scatt }}$. If we assume all of the diffracted light is considered removed from the incident signal (as is the case in the far-field), then diffraction removes an amount of energy corresponding to an effective cross section equal to $G_{\text {scatt }}$ as well. Therefore, the total energy removed from the incident plane wave corresponds to a cross section equal to twice the geometric area of the particle (van de Hulst, 1957).

This has important implications for occultation data. During an occultation, a single ring particle will remove an amount of energy equal to twice that which would be expected for a particle of a given geometric area. This results in an optical depth measurement that is twice as high when compared with the optical depth due to blocked (absorbed/back-scattered) light only. Therefore an individual particle in the geometric optics regime has an extinction efficiency, $Q_{\text {ext }}$, that is equal to 2. However, during observations of Saturn's rings, the light that has been diffracted out of the instrument's FOV by one particle can be replaced by near-by particles that are diffracting light into the FOV, changing the effective extinction efficiency to a value somewhere between 1 and 2. Furthermore, because the Sun is not a point source, the same particle can diffract light from different parts of the Sun into the FOV, replacing in part, or entirely, the light diffracted out of the FOV (Cuzzi and Pollack, 1978; French and Nicholson, 2000).

The effective extinction efficiency is used to account for the light removed and replaced by the rings simultaneously. In this work, we model the signal removed from the FOV by absorption/scattering/diffraction independently from the signal added to the FOV by diffraction. Because the particle sizes included in the model are large enough to be in the geometric optic regime, $Q_{\text {ext }}$ is always assumed to be equal to 2 when modeling the signal removed from the FOV by the particles.

\subsection{Application to Saturn's Rings}

We consider the sub-millimeter particle population in Saturn's F ring to be well-described by the "classical" ring model, which assumes the particles that make up the ring are uniformly distributed in a loosely-packed, extended layer that is many-particles thick (Cuzzi et al., 2009). In this model, the interaction between light and the particles is treated as a radiative-transfer 
problem. We model the particle size distribution of the ring by a power-law defined as (Cuzzi et al., 2009)

$$
n(a) d a=C a^{-q} d a, a_{\min } \leq a \leq a_{\max }
$$

where $n(a)$ is the number of particles with a radius in the range $[a, a+d a]$, $d a$ is the infinitesimal increment in $a, C$ is a constant, and $a_{\min }$ and $a_{\max }$ are the minimum and maximum particle sizes in the distribution, respectively. Power-law size distributions have successfully described the ring material in other analyses (e.g., Zebker et al. (1985); Showalter et al. (1992); French and Nicholson (2000); Cuzzi et al. (2009); Charnoz et al. (2009)), though we note that Hedman et al. (2011) required a broken, 2-component power-law model to fit VIMS observations of the F ring. A power-law with an index of $q \sim 3.5$ is a typical distribution for a population that has evolved through collisional disruption (Dohnanyi, 1969).

Assuming the incoming light is diffracted only one time, the intensity of the diffracted signal, $I_{1}(\theta)$, at a given wavelength is written explicitly as (Cuzzi et al., 2009)

$$
I_{1}(\theta)=\frac{I_{i} e^{-\tau_{n} / \mu_{0}}}{4 \pi \mu_{0}} \int_{a_{\min }}^{a_{2}} \pi a^{2} P(\theta, a) n(a) d a
$$

for

$$
P(\theta, a)=\left[\frac{2 J_{1}(k a \sin \theta)}{\sin \theta}\right]^{2}
$$

where $P(\theta, a)$ is the phase function for Fraunhofer diffraction, $k=\frac{2 \pi}{\lambda}, J_{1}$ is the Bessel function of the first kind and order $1, a_{2}$ is the maximum particle size contributing the diffracted signal, $\theta$ is the scattering angle, and $\mu_{0}=$ $\sin \left|B_{S U N}\right|$ where $B_{S U N}$ is the angle between the ring plane and incident radiation (ring opening angle). The $B$ values for each solar occultation are listed in Table 1. $\tau_{n}$ is the normal optical depth of the ring (the optical depth as viewed perpendicular to the ring) and is defined in Equation $5 . I_{i}$ is the intensity of the incident light. The phase function is normalized such that $\int P(\theta) d \Omega=2 \pi \int_{0}^{\pi} P(\theta) \sin \theta d \theta=4 \pi$ (French and Nicholson, 2000).

The normal optical depth of the ring is related to the particle size distribution (Cuzzi, 1985; Cuzzi et al., 2009) as

$$
\tau_{n}=\int_{a_{\min }}^{a_{\max }} \pi a^{2} Q_{e x t} n(a) d a
$$


$\tau_{n}$ can be derived from observations; however, in this work we treat $\tau_{n}$ as an input parameter to determine how the optical depth affects the model signal. Substituting Equation 2 into Equation 5, we can solve for C:

$$
C=\frac{\tau_{n}}{\int_{a_{\min }}^{a_{\max }} \pi Q_{e x t} a^{2-q} d a} .
$$

Equation 6 can be substituted into Equation 2, which is then substituted with with Equations 4 and 5 into Equation 3. In order to solve this computationally, we approximate the integral over the Bessel function as a summation and solve for the intensity of the diffraction signature as

$$
I_{1}=\frac{I_{i} e^{-\tau_{n} / \mu_{0}}}{\mu_{0}} \sum_{j=0}^{N_{\max }}\left[\frac{J_{1}\left(k a_{j} \sin \theta\right)}{\sin \theta}\right]^{2} C a_{j}^{2-q} \Delta a_{j}
$$

where $j$ is the $j^{\text {th }}$ element in a range of particle sizes from $a_{\min }$ to $a_{2}, a_{N_{\max }}$ $=a_{2}$, and $\Delta a_{j}$ is the incremental increase in the range $\left[a_{j}, a_{j+1}\right]$. Throughout this work, we assume the maximum particle size in the particle size distribution of the $\mathrm{F}$ ring envelope is $a_{\max }=1 \mathrm{~mm}$. The parameters $a_{\min }, q$, and $\tau_{n}$ are inputs into the model, and so Equation 7 can be solved explicitly. In the following section we describe how we incorporate these equations in the computational model.

We exclude other forms of scattering by the rings, such as reflection and refraction, from contributing to the total observed signal. Ignoring other forms of scattering is justified; the rings are highly-absorbing at UV wavelengths (Bradley et al., 2010), so scattering through reflections during occultations contributes very little to the observed signals.

Because additional scattering contributes weakly to the overall signal, we ignore multiple scattering in the traditional sense. We do consider the effects of a secondary or tertiary diffraction event, which we call multiple diffraction; however, as shown by Becker (2016), due to the very low average optical depths $\left(\tau_{\text {avg }} \leq 0.07\right)$ across the $\mathrm{F}$ ring $(\sim 300-600 \mathrm{~km})$, multiple diffraction would contribute little to the observed signal.

\section{Spectral Analysis}

In order to apply the equations described in Section 4 to models of Saturn's rings, we must assume that the particles are in the geometric optics regime $(a>>\lambda)$. This is typically true for a size parameter of $X \geq 20$, where 
$X=\frac{2 \pi a}{\lambda}$ (van de Hulst, 1957). For the EUV wavelengths of $56-118 \mathrm{~nm}$, this corresponds to particles with $a=178-375 \mathrm{~nm}$. Therefore, if the majority of the particles have radii larger than $\sim 0.2 \mu \mathrm{m}$, then we can model the diffracted signal with the Fraunhofer diffraction equations described above.

We can verify that we are in the geometric optics regime by looking at the F ring occultation light curve at various wavelengths. Bosh et al. (2002) measured the equivalent depth (the area under the curve) of the $\mathrm{F}$ ring in HST data from $0.27-0.74 \mu \mathrm{m}$ and found no variations in the equivalent depth with wavelength. If there were a significant number of particles that were not within the geometric optics regime, then the equivalent depth of the $\mathrm{F}$ ring would vary with wavelength.

We investigate any variation in the $\mathrm{F}$ ring light curve profile at different wavelengths and find no wavelength dependency for the properly aligned solar occultations. In Figure 3 we show four examples, including two solar occultations with no UVIS diffraction signatures (Rev 59 (I) and Rev 65 (E)) and two occultations with diffraction signatures (Rev 43 (E) and Rev 55 (E)). We bin the spectrum into 5 bins, summing the signal over $12 \mathrm{~nm}$ in wavelength. Though the data is somewhat noisy, there is no indication of a change in equivalent depth at each wavelength. Therefore, we conclude that the bulk of the particles in the F ring are larger than $0.2 \mu \mathrm{m}$ in size.

We also search for variations in the spectra by comparing the signal found in the F ring core with the unocculted solar signal. Figures 4 and 5 show the regions used for the spectral comparison. We subtract the spectral signal of the unocculted Sun, $I_{0}$ from the signal at the $\mathrm{F}$ ring core, $I_{\text {core }}$ and divide by the unocculted solar signal. We do the same for the diffraction signature if available. The resulting spectral comparisons for all of the occultations look very similar: they are flat with no obvious indication of variation in signal as a function of wavelength. This is also true for the diffracted signal. We fit a line of the form $y=A+B x$ to the comparative signals and include a list of the best-fit parameters A, B, and their $1 \sigma$ uncertainties in Table 4 . We note, however, that our analysis of the spectral variations through the $\mathrm{F}$ ring particles is difficult since the projected width of the $\mathrm{F}$ ring is smaller than that of the Sun, so the solar spectrum from direct solar light is also observed while looking for any spectral signatures due to transmission or absorption by the F ring particles at a given wavelength. At UV wavelengths, however, Saturn's rings are extremely dark due to significant absorption of the light by the water ice particles, so we would not expect to see any features at EUV wavelengths. Further, the quality of the spectral data limits our ability to see 
any slope or features in the data as a function of wavelength and therefore we cannot make any additional qualifications about the particle size distribution of the $\mathrm{F}$ ring.
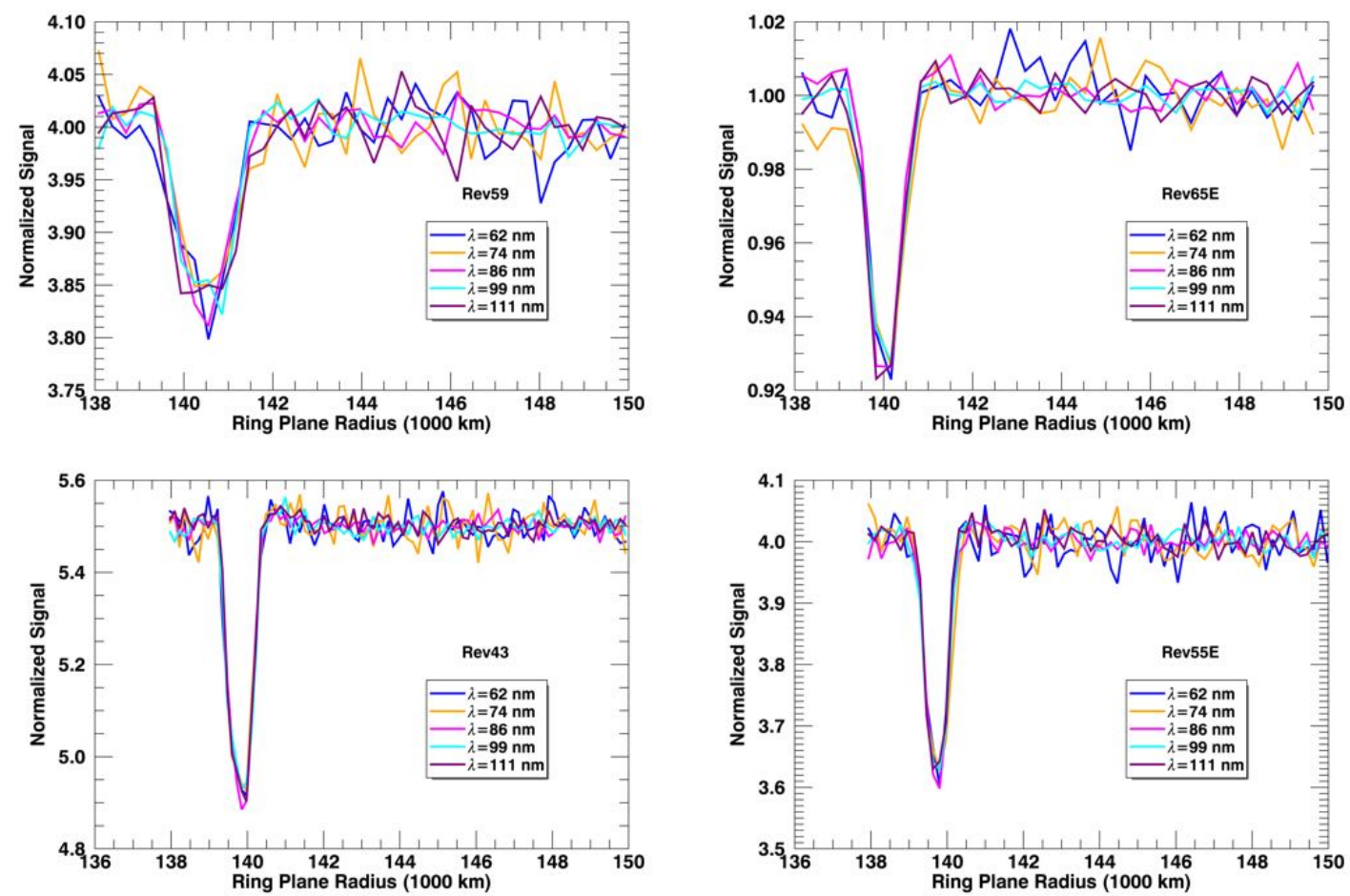

Figure 3: The total signal at 5 different wavelengths as a function of radius for four solar occultations. Each wavelength bin is the sum of $12 \mathrm{~nm}$ in wavelength and is represented by the median value of wavelength in the legend. Two of these occultations show diffraction signatures (Rev 43 and Rev 55 (E), while the other two do not. The shape of the light curve, including the width and depth of the light curve, do not vary significantly with increasing wavelength.

\section{Computational Model}

We have developed a computational model that replicates the geometry of each solar occultation and models both the attenuated signal and the diffracted signal that would be produced by an $\mathrm{F}$ ring of a given width $(W)$, 

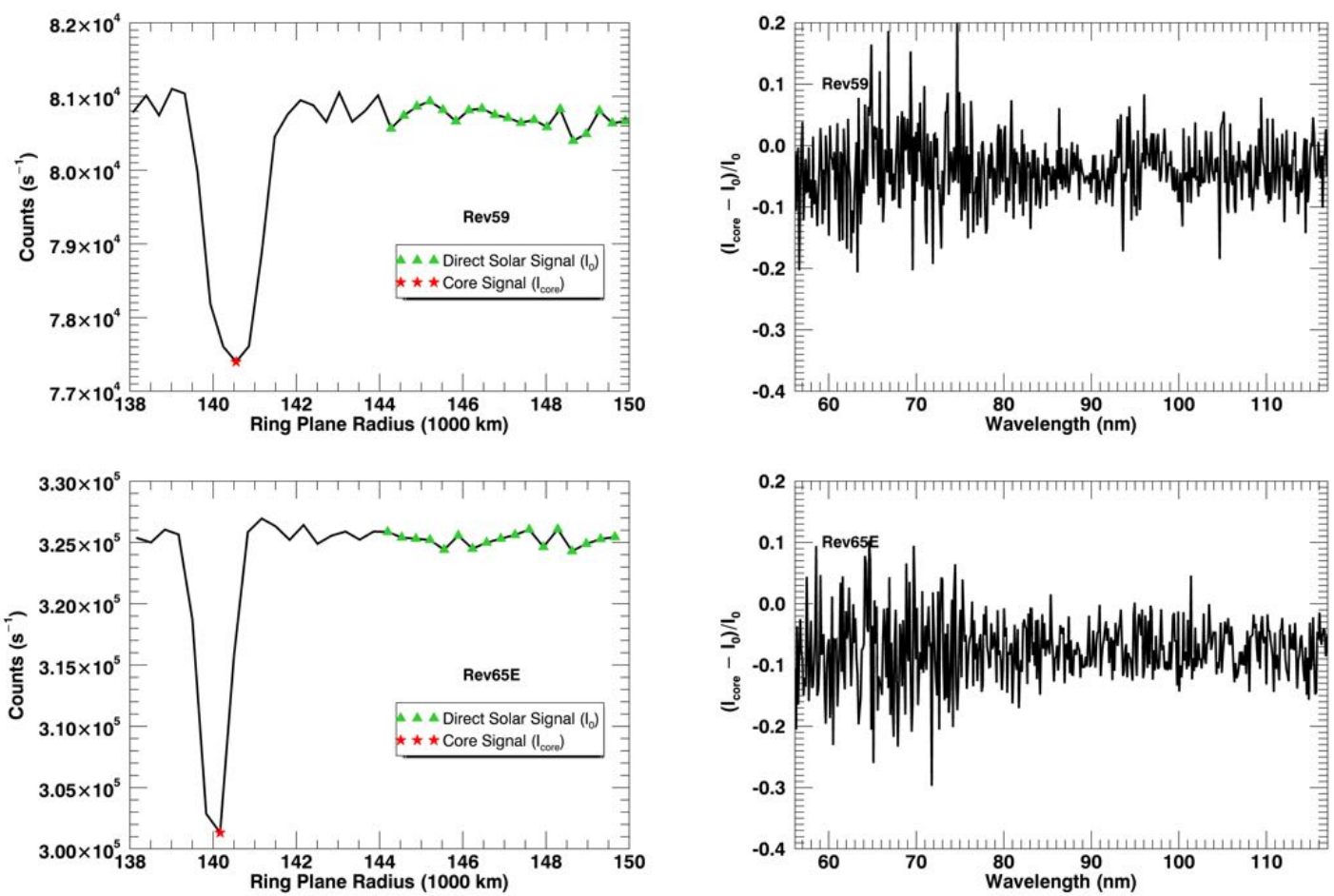

Figure 4: Column 1 displays the the solar occultation data without UVIS diffraction signatures and the regions used for the spectral analysis shown in Column 2. Green triangles mark the unocculted solar signal $\left(I_{0}\right)$ used for this analysis and the red star marks the $\mathrm{F}$ ring core $\left(I_{\text {core }}\right)$. In Column 2 we show the differences in the intensity as a function of wavelength by subtracting the mean spectral signature from the unocculted solar signal (green triangles) from the spectra in the F ring core (red star) and then dividing by the unocculted solar signal. The Rev $65 \mathrm{E}$ occultation has been binned by 4 data points. 

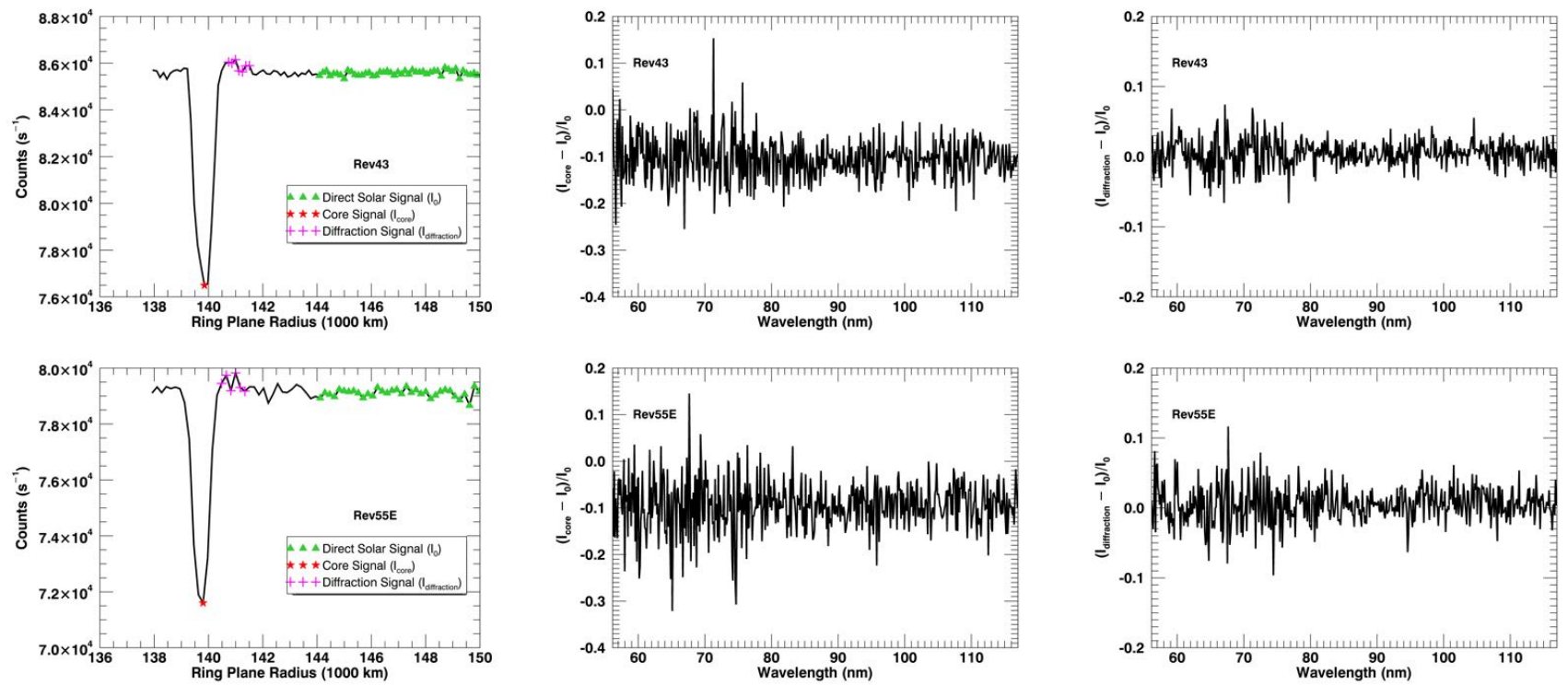

Figure 5: Column 1 displays the the solar occultation data with UVIS diffraction signatures and the regions used for the spectral analysis shown in Column 2. In addition to the unocculted solar signal (green triangles) and signal through the F ring core (red star), we also analyze the diffraction signature (magenta ' + ') . Column 2 shows the normalized difference in signal between the unocculted solar spectrum and the spectrum at the $\mathrm{F}$ ring core as shown in Figure 4. Column 3 shows the difference between the spectral signature from the data points near or in the diffraction signal and the unocculted solar signal, normalized by that unocculted solar signal. These plots show little variation in signal as a function of wavelength. 
average optical depth $\left(\tau_{\text {avg }}\right)$, and a particle size distribution defined by $a_{\text {min }}$ and $q$, with $a_{\max }=1 \mathrm{~mm}$. We run a suite of models to cover the phase space for each of these four parameters $\left(W, \tau_{\text {avg }}, a_{\text {min }}\right.$ and $\left.q\right)$. Table 6 shows the parameters used to model the solar occultations.

We use SPICE kernels and routines made available by NASA's Navigation and Ancillary Information Facility (NAIF, 2015) to determine the geometry of each occultation, including the position of the spacecraft and the pointing of the instrument. We build a model of the UVIS FOV (Section 6.1) and determine where the rings and the Sun are located in each time step of the model. We then apply the equations described in Section 4 to determine the total signal expected from a ring given the initial parameters. We describe the model in more detail in the following sections.

\subsection{Building the FOV}

We model the UVIS solar port field of view as a set of discrete elements that cover the angular length and width of the FOV. The size of the FOV elements (or, the number of elements used) is optimized to adequately capture variations in the intensity of the diffraction signal without being computationally expensive. Figure 6 shows how the intensity of the phase function from diffracted light varies with angular separation from the light source for each particle size regime. From this figure, we find that for particles up to 1 millimeter in size, the intensity of the scattered light will be constant for scattering angles less than $10^{-5}$ radians. We consider this angle, which defines the diffraction pattern's first minimum and before which the intensity of the signal is nearly constant, to be the critical scattering angle. We do not require a model with a higher resolution than the critical scattering angle. Additionally, with the exception of a few time-steps in each model when the ring is occulting the Sun, the angular separation between the ring particles and the Sun are much greater than the critical scattering angle, so even lower spatial resolutions can be applied.

To minimize the computational time required for each model, we produce a low-resolution virtual FOV with square elements with an angular size of $10^{-5} \times 10^{-5}$ radians for the entire FOV, and a higher-resolution $\left(8 \times 10^{-6}\right.$ radians), smaller FOV that encompasses the region of the Sun and the F ring for time steps when the ring is occulting (or nearly occulting) the Sun. The slight increase in the resolution is to enhance the spatial resolution of the Sun and the ring during the occultation. 
Following the model described by Becker et al. (2016), for each time step of the occultation, we determine the ring-plane-intercept radius for each element in the FOV. Because the F ring is eccentric, we must calculate the radial location of the ring at the inertial longitude of the UVIS boresight's ring-plane intercept. We use the $\mathrm{F}$ ring orbital parameters (Table 5) described by Albers et al. (2012) to calculate the ring plane radius of the $\mathrm{F}$ ring. We then flag all elements that are within a distance of $\frac{1}{2} W$ (the model ring's width) from the core as "ring elements".

At Saturn, the Sun subtends an angle of $\sim 1 \mathrm{mrad}$. Therefore, unlike the stellar occultation model in Becker et al. (2016), we cannot approximate the light source as a point source. Light emanating from all parts of the Sun will be diffracted by the ring particles, so we must account for the Sun's angular size. We reconstruct the Sun's position in the FOV (we do not assume it is centered in the FOV, see Section 6.3) and calculate its angular size for each occultation assuming a solar diameter of 1,391,000 km. We build the slightly higher-resolution FOV $\left(8 \times 10^{-6}\right.$ radian elements $)$ in a region encompassing the Sun and flag all elements coincident with the Sun as "Sun elements".

\subsection{Model Signal}

\subsubsection{Attenuated Signal}

The model signal is comprised of two parts: the transmitted signal and the diffracted signal. During most of the occultations, the geometry is such that the angular extent of the F ring is smaller than that of the Sun, so only part of the direct solar signal is attenuated. We determine the total attenuated, transmitted signal at each time step by measuring the fraction of the Sun that is covered by ring material. We divide the total area of the elements that are simultaneously Sun elements and ring elements, $A_{\text {occ }}$, by the total area of the Sun elements $A_{\odot}$. Assuming an opaque ring, the signal from the unocculted part of the Sun during the occultation can be calculated as

$$
I_{\text {unocculted }}=I_{0}\left(1-\frac{A_{o c c}}{A_{\odot}}\right) .
$$

If such a ring were covering $10 \%$ of the Sun, the observed signal would be $I_{\text {unocculted }}=0.9 I_{0}$, or $90 \%$ the total solar signal. However, the $\mathrm{F}$ ring is not opaque, so we must include the attenuated light that passes through the 


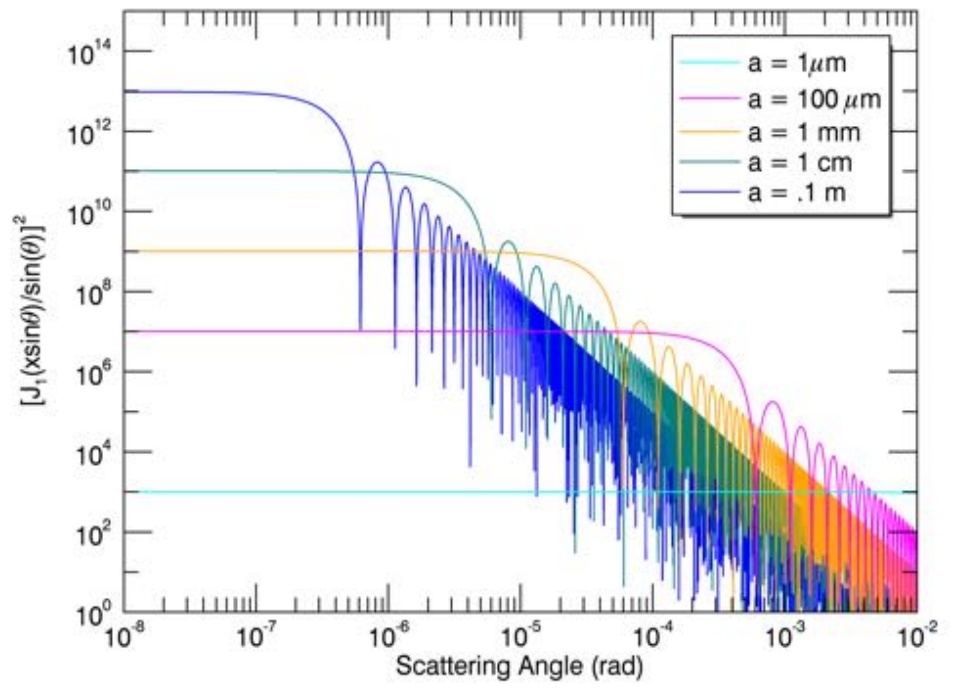

Figure 6: The phase function $\mathrm{P}(\theta)$ for diffracted light as a function of scattering angle, $\theta$. Larger particles diffract light with a higher intensity but over a smaller range of scattering angles. There is a critical scattering angle for each particle size that defines the diffraction signal's first minimum (Equation 1), before which the intensity of the signal is nearly constant. 
rings. We apply the equation for normal optical depth, $\tau_{n}$, with negligible background (Eq. 9) from Colwell et al. (2010)

$$
\tau_{n}=\mu \ln \left(\frac{I}{I_{0}}\right)
$$

where $I$ is the measured attenuated signal, and $\mu$ is the sine of the ring opening angle $B$, to only the portion of the Sun being occulted:

$$
I_{\text {attenuated }}=I_{0} e^{-\tau_{\text {avg }} / \mu}\left(\frac{A_{o c c}}{A_{\odot}}\right) .
$$

The total signal due to attenuation by the ring is then computed as the sum of Equations 8 and 10:

$$
I_{\text {transmitted }}=I_{\text {unocculted }}+I_{\text {attenuated }} .
$$

At EUV wavelengths, the distribution of the Sun's intensity is complex (Amblard et al., 2008). Including the nuances of the signal variation from the Sun is beyond the scope of this paper. Instead, we assume a projected homogenous solar disk with a diameter of 1.391 million $\mathrm{km}$. We note possible effects on the model results due to these assumptions in Section 7.

\subsubsection{Diffracted Signal}

The contribution to the signal from the light diffracted by the ring particles is dependent on the scattering angle between those particles and the light source. Because the Sun is not a point source, we must calculate the scattering angle between each ring element and every Sun element. For each ring element, we apply Equation 3 to calculate the amount of light diffracted by particles in that region due to the solar signal emanating from each of the Sun elements, based on the scattering angle between the elements, and sum the signal to determine the overall diffracted light by the particles in that region of the ring. We repeat this process for all of the ring elements. Next, we sum over the resulting signal intensity of each element and normalize by the solid angle of the ring element. Finally, we combine the total diffracted light at each model point with the attenuated signal calculated in the previous section to determine the final model signal that is directly compared with the data. The final model accounts for the transmitted solar signal that has been attenuated through absorption and scattering by ring particles as well as the additional solar signal caused by diffraction from different parts of the Sun and surrounding particles into the field of view. 


\subsection{FOV Sensitivity}

The UVIS Solar Port field of view is not uniformly sensitive to incoming photons. We have conducted a full spatial analysis of the solar port FOV, details of which can be found in Becker (2016). Figure 7 is a map of the signal sensitivity. We use this map, and the location of each element within the FOV, to determine by how much we reduce the theoretical signal observed by that element. The FOV map is described by an X-Y coordinate system in the UVIS detector frame (not that defined by Cassini SPICE kernels), where the X-axis is the direction of increasing wavelength (across the UVIS slit) and the $\mathrm{Y}$-axis is perpendicular to that (along the UVIS slit).

As a result of this position-dependent signal strength, we must account for the fact that the intensity of the unocculted signal, $I_{0}$, is location-dependent. We find the Sun's location in the FOV and extrapolate the true signal of the incident sunlight based on the measured signal. This value, $I_{i}$, is what the signal would have been if the FOV were to have uniform sensitivity. It is calculated by

$$
I_{i}=I_{0} / S
$$

where $S$ is the percent reduction of the solar signal due to the non-uniform sensitivity of the instrument FOV. When we calculate the diffraction signal, we assume the incident intensity of the light is $I_{i}$ on all particles. We reduce the final model signal from each FOV element by a value $S$ determined by the location of the model element within the instrument FOV.

We implement one additional step for calculating $S$ for the offset Rev 9 occultation. If we assume the C-kernel that describes the instrument pointing is correct for this occultation, the resulting decrease in solar signal is not consistent with the observed 2,000 counts described in Section 1. Since the Sun is at the very edge of the FOV, even an extremely small offset from the C-kernel position can cause a drastic change in counts, unlike when the Sun is more closely centered in the FOV (Fig. 7). Therefore, we assume a solar count signal close to that observed in the next solar occultation (Rev43: 85,000 counts per second) and shift the Sun along the X-axis of the model FOV such that the resulting unocculted signal is the observed 2000 counts per second. We assume the slight offset is in the X-direction because the dropoff in signal is much steeper along the $\mathrm{X}$-axis (corresponding almost entirely to the percentage of the Sun visible to the instrument) compared

with the dropoff along with y-direction. We find that an adjustment in the 
x-direction of 0.139 mrads decreases the solar signal to that observed in the Rev 9 occultation, a minor offset in the C-kernel pointing, compared with the $1 \mathrm{mrad}$ adjustment required if the shift were along the $\mathrm{Y}$-axis. This offset is well within the pointing errors of the reconstructed C-kernel.

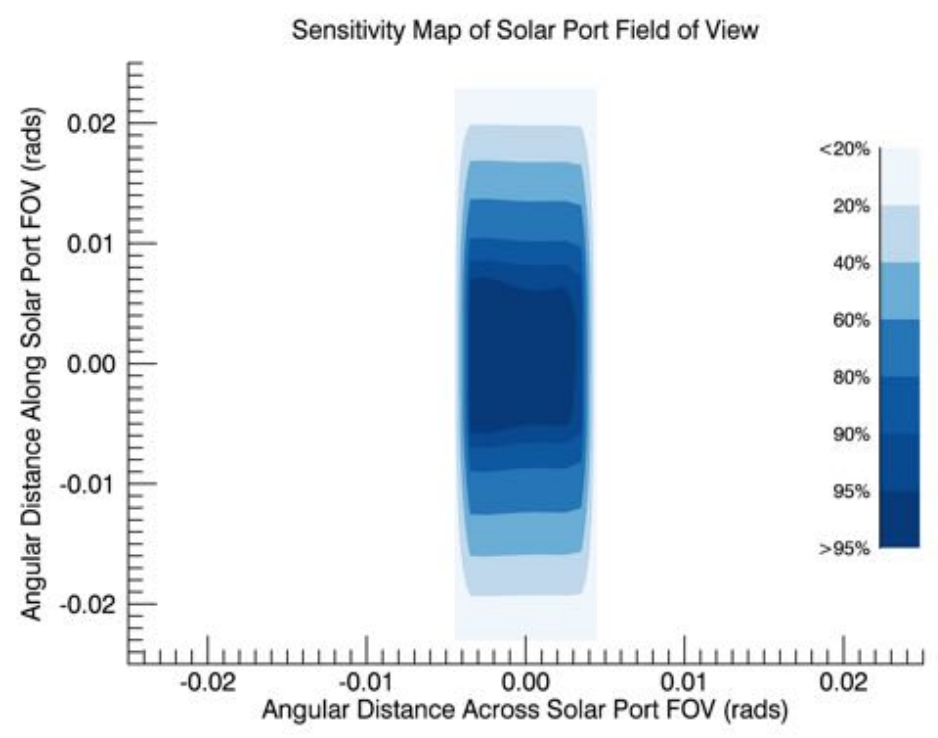

Figure 7: The characterization of the solar port field of view sensitivity. Dark blue to light blue indicates the percent decrease in sensitivity relative to the maximum signal, as shown in the scale. The $\mathrm{x}$ - and $\mathrm{y}$ - axes correspond to the $\mathrm{x}$ - and $\mathrm{y}$-axes of the solar port FOV. This map was created through the analysis of multiple calibration scans executed by Cassini. Details can be found in Becker (2016).

\subsection{Model Parameters}

We use our forward-modeling approach to reproduce the observed UVIS light curves. We explore the parameter space for four variables that we input into the model: the width of the ring $(\mathrm{W})$, the average optical depth of the $\mathrm{F}$ ring $\left(\tau_{\text {avg }}\right)$, the minimum size in the particle size distribution $\left(a_{\min }\right)$ and the slope of the power-law size distribution $(q)$. While there are variations in the optical depth of the F ring, the ring itself is not resolved in the solar occultations due to the large projected size of the Sun. We therefore assume a constant, average optical depth across the ring. This can be a limitation of the model; however, we do briefly explore the more realistic scenario of an 
F ring core with a higher optical depth embedded in a broader envelope of particles with a different size distribution in Section 6.4.4. In the following sections, we discuss the input parameters.

\subsection{1. $a_{\min }$ and $q$}

We use constraints on the particle size distribution of the $\mathrm{F}$ ring from previous observations to guide our model inputs. The Voyager RSS occultation (Tyler et al., 1983) did not detect the $\mathrm{F}$ ring envelope at either of its wavelength bands $(3.6 \mathrm{~cm}$ and $13 \mathrm{~cm}$ ), placing an upper limit of $\sim 1 \mathrm{~mm}$ on the $\mathrm{F}$ ring particles outside of the core. A lower bound of $\sim 1 \mu \mathrm{m}$ is set by the Cassini VIMS data, which show no spike in the F ring optical depth at a wavelength of 3.1 microns, indicating a depletion of sub-micron-sized particles (Hedman et al., 2011). They find that a broken power-law size distribution for the $\mathrm{F}$ ring is required to fit the data: a shallow distribution for particles $\leq 10 \mu \mathrm{m}$, and a value of $q=3.5$ for the particles with radii larger than $10 \mu \mathrm{m}$ and smaller than an assumed $1 \mathrm{~mm}$ maximum particle size. Observations from the HST occultation of the star GSC5249-01240 also led Bosh et al. (2002) to conclude that no significant population of sub-micron-sized particles exist in the $\mathrm{F}$ ring region.

Based on these observations, we place a maximum particle size, $a_{\max }=1$ $\mathrm{mm}$, for all models, and we vary our minimum particle size $a_{\min }$ between $1 \mu \mathrm{m}$ and $500 \mu \mathrm{m}$ in radius. We also test a series of $q$ values, from $q=2.7$ to $q=4$. See Table 6 for the values used for $a_{\min }$ and $q$. We run the same suite of particle size distribution parameters for every occultation and every input for $W$ and $\tau_{\text {avg }}$ of the ring.

Figure 8 shows how varying the particle size distributions affects the resulting diffraction signal during a solar occultation, and therefore the observed light curve. The smaller particles diffract light at a larger angular extent, so a ring with small (tens of microns) particles will begin diffracting light before the ring has even begun occulting the Sun and after the occultation has finished (see blue stars in Figure 8). When we then include the direct, attenuated signal (red ' $\mathrm{X}$ '), we see that the final light curve (solid blue line) for a model with a small minimum particle size has a deep dip and small increase over the unocculted signal (diffraction "ears") immediately before or after the dip. The larger particles diffract the incident sunlight over a narrower cone (green squares). The diffracted signal effectively works to decrease the depth of the light curve dip by replacing much of the sunlight that is removed by the particles through absorption, 
reflection and diffraction. The result (solid green line) for a model with a relatively larger minimum particle size is a shallower dip, with little to no indication of diffraction signals on either side of of that dip.

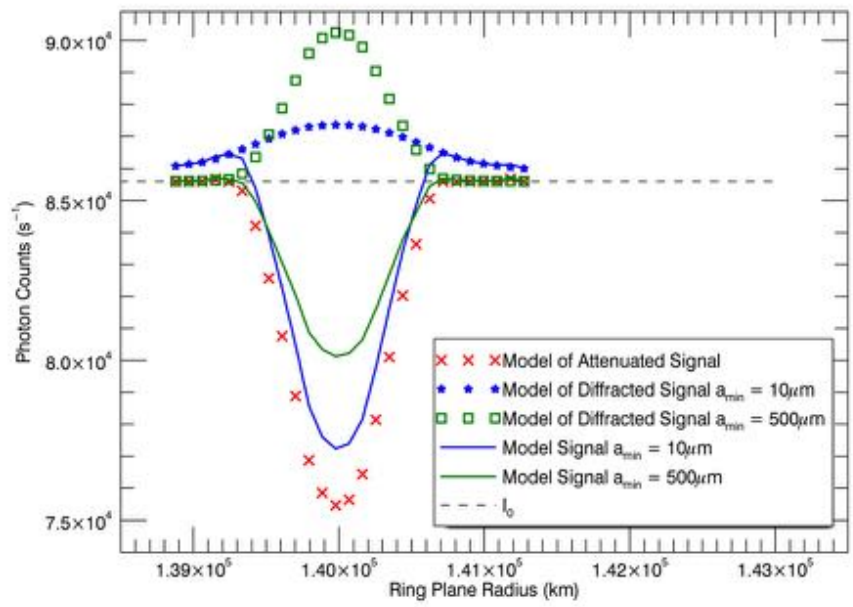

Figure 8: Solar occultation model for two different minimum particle sizes. All other parameters are the same. The model with $a_{\min }=10 \mu \mathrm{m}$ (blue stars) produces a diffraction signal that is broad, while the model with $a_{\text {min }}=500 \mu \mathrm{m}$ (green squares) produces a diffraction signal that has a higher intensity, but more narrowly distributed. The red ' $\mathrm{X}$ ' shows the modeled attenuated signal caused by the removal of sunlight as the ring occults the Sun, which is independent of the particle size (it only depends on the width and optical depth of the ring). When the diffraction signals are combined with the attenuated signal, we find that the final model signal for the model with $a_{m i n}=10 \mu \mathrm{m}$ (blue solid line) has a deeper light curve and small signal "diffraction ears" before and after the signal dip. The final model with $a_{\text {min }}=500 \mu \mathrm{m}$ (green solid line) has a shallower dip and no detectable additional signal above the baseline unocculted solar signal. This example uses the geometry of Rev 43 for a model ring with $\mathrm{W}=500 \mathrm{~km}$ and $\tau_{\text {avg }}=0.05$.

\subsubsection{Ring Width W}

Initially we expected to model the traditional $\mathrm{F}$ ring core, a narrow ring $\sim 50 \mathrm{~km}$ across. However, we quickly found that models with such a small 
width could not reproduce any data set. As a result, we recognized that the light curve was reflective of the much broader $\mathrm{F}$ ring envelope, since no models of a thin ring could match the data. Therefore we test models with a $\mathrm{F}$ ring widths equal to $100 \mathrm{~km}, 250 \mathrm{~km}, 350 \mathrm{~km}$, and $500 \mathrm{~km}$. We also run models for $W=580 \mathrm{~km}$ to test the value of the ring width derived by French et al. (2012). Figure 9 illustrates how the model is affected by the ring width. The size of the ring has a critical effect on the overall light curve: the ring width determines how much of the Sun is covered by the particles and therefore how much light is extinguished during the occultation. A wider ring with the same optical depth as a thinner ring provides more particles to diffract light throughout the occultation, producing the observed peaks above the baseline, direct solar signal (diffraction ears) on either side of the dip in the light curve.

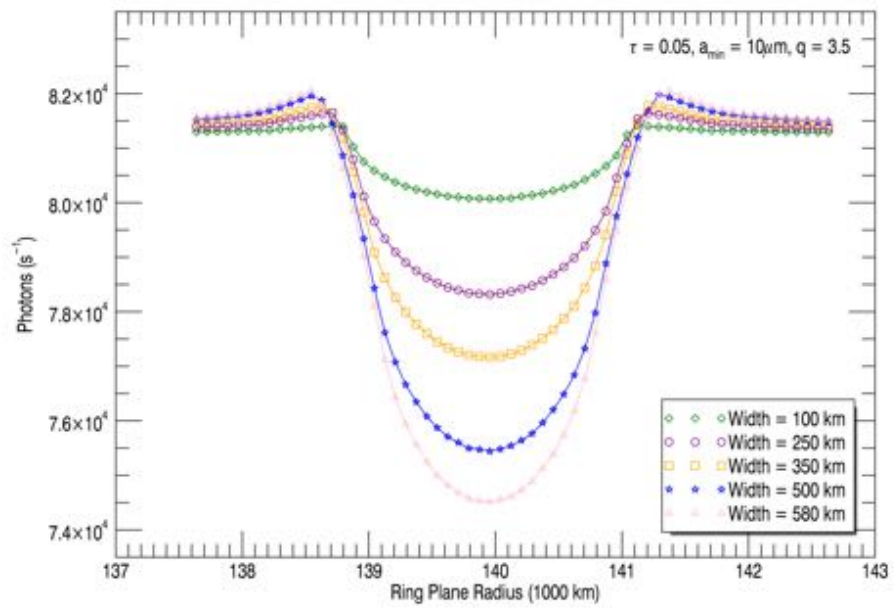

Figure 9: Solar occultation model for varying $\mathrm{F}$ ring widths. When all other parameters are constant, the width affects how much of the Sun is covered during the occultation, reducing the light curve dip, and the availability of more particles to diffract light during the occultation, creating the observed diffraction ears adjacent to the light curve dip.

\subsubsection{Average Optical Depth $\tau_{\text {avg }}$}

For all models of the rings, we use three optical depths: $\tau_{\text {avg }}=0.03,0.05,0.07$. These values were determined based on initial modeling. As we began analyzing results, we found that the models with larger widths typically produced 
better fits to the data, so we included two additional average optical depths, $\tau_{a v g}=0.02$, and 0.035 , for ring models with $W=500,580 \mathrm{~km}$.

\subsubsection{Embedded Core}

We briefly explore the effects of including a narrow $\mathrm{F}$ ring core of relatively higher optical depth embedded within the wide $\mathrm{F}$ ring envelope in our modeling efforts. Figure 10 is an example of the resulting model. We model the two rings entirely independently, then combine their diffraction and attenuated signals. Figure 10 shows, as expected, that the overall depletion of counts increases when a core of higher optical depth is included in the model. The shape of the dip is also affected by the embedded core.

Although such a model is feasible, adding an F ring core with its own set of parameters adds several additional free parameters. Furthermore, the higher optical depths in the F ring core would necessitate a model that includes multiple scattering to properly model the diffraction signals produced in this region of the $\mathrm{F}$ ring. For this paper, we focus on a uniform $\mathrm{F}$ ring with a given $\tau_{\text {avg }}$ that best accounts for the higher optical depth in the core as well as the lower optical depth in the diffuse F ring envelope.

\subsection{Occultation Timing}

An additional uncertainty in the models is the exact timing of the occultation. Since the evaluation of the models is based on a direct comparison of the model light curve and the data at each time step of the observation, a small offset of the ring-encounter time can drastically affect the resulting model's fit to the data. This is especially true for observations with longer integration periods. Although we calculate the position of the $\mathrm{F}$ ring based on its orbital parameters (Section 6), variability of the F ring core's radial distance from Saturn can result in a model where the occultation begins slightly earlier or later than observed. To account for this issue, we run 2-3 models with slightly different start times for most occultations with integration periods $>1$ second and use the model that provides the best fit to the data. Figure 11 shows identical models of the Rev 55 (I) solar occultation that have occultation start times with 1 second differences. This observation has a 4 second integration period and so the three models capture different parts of the light curve as the ring occults the Sun. 


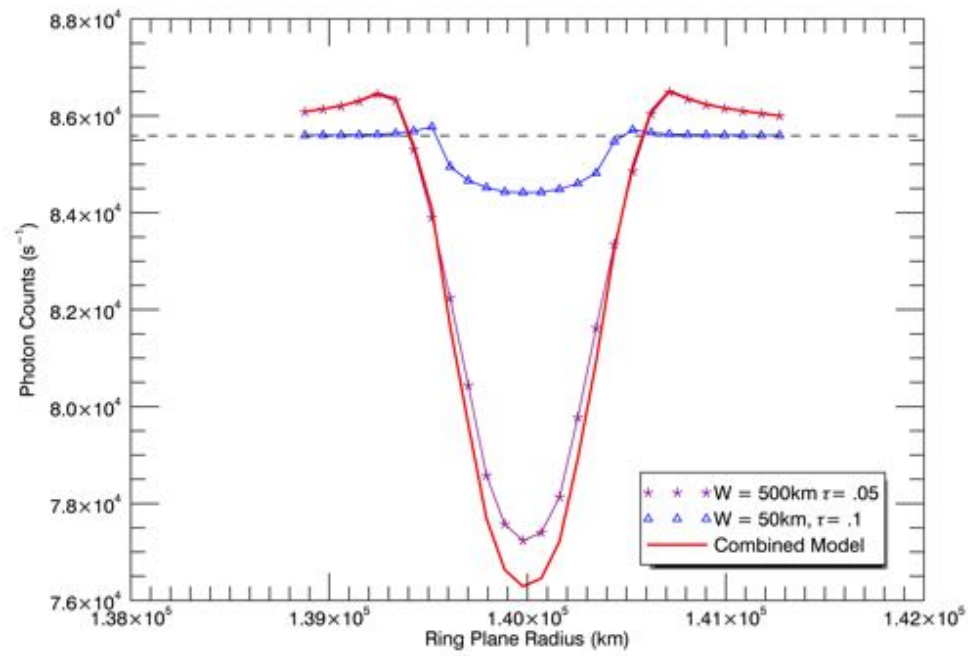

Figure 10: Solar occultation model of an $\mathrm{F}$ ring with a core embedded in a larger envelope of particles. The input parameters of the core are: $a_{\min }=100 \mu \mathrm{m}, \tau_{\text {avg }}=0.10, W=50$ $\mathrm{km}$. The parameters for the envelope are: $a_{\min }=10 \mu \mathrm{m}, \tau_{\text {avg }}=0.05, W=500 \mathrm{~km}$. For both rings, $q=4.0$ and $a_{\max }=1 \mathrm{~mm}$. As expected, embedding a narrow core decreases the model counts at the time that the core occults the Sun, but does not contribute much to creating a signal above of the unocculted baseline solar signal. 

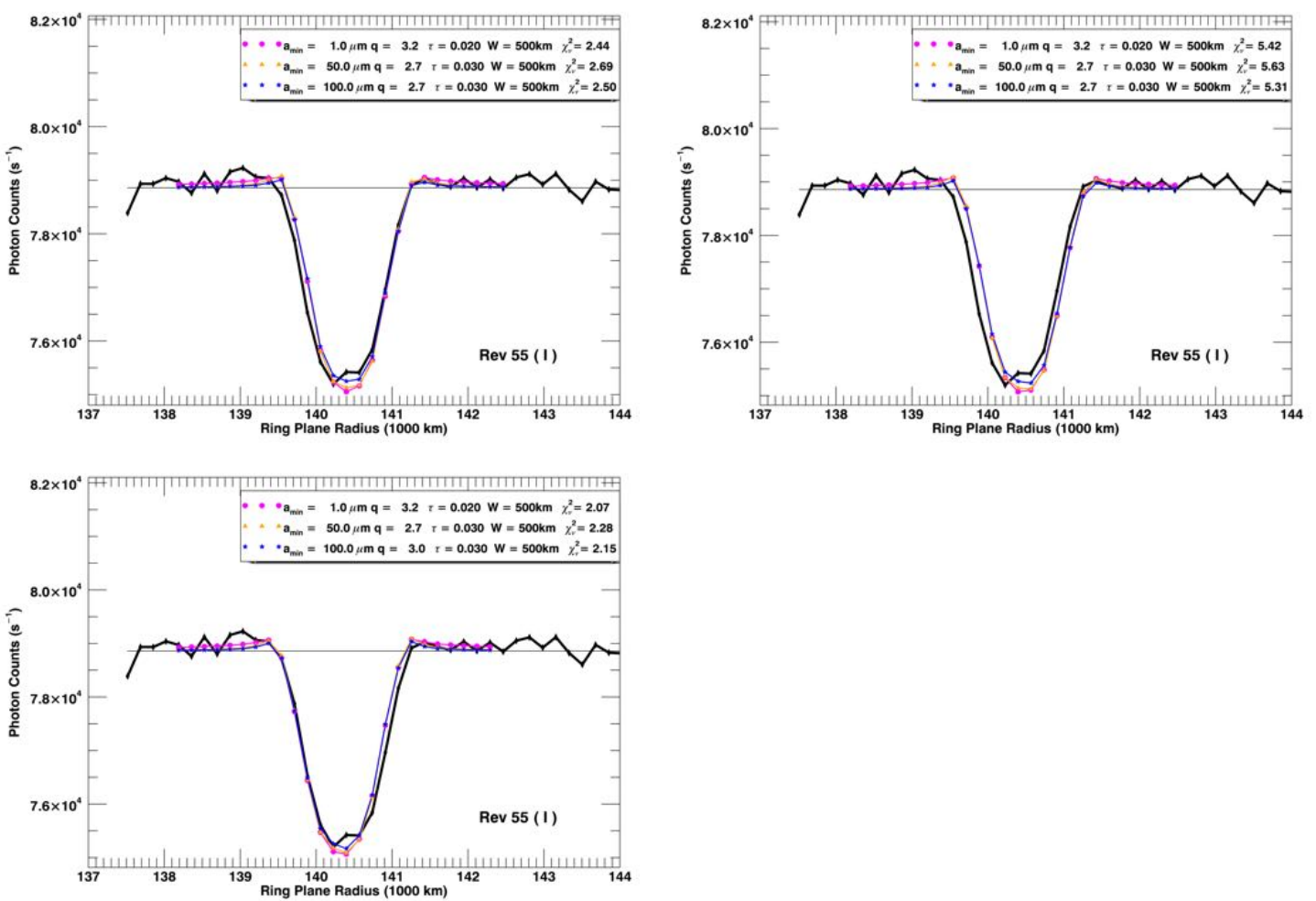

Figure 11: Three identical models of the Rev 55 (I) solar occultation, but with 1 second offsets in the occultation start times. Because the observations have a 4-second integration period, a slight offset in the timing of the model point acquisition compared with the data point acquisition results in a different sampling of the ring. We run the suite of models 2-3 times for each occultation with integration periods longer than 1 second and use the models with the best visible and $\chi_{\nu}^{2}$ fits. For the case of Rev 55 (I), we use the occultation start time that produces the third plot in this figure. 


\section{Results}

We produce light curve models of each occultation for every combination of input parameters described in Section 6.4. We evaluate each model by performing a direct comparison of each data point as a function of ring location and the corresponding model points and calculating the reduced $\chi^{2}\left(\chi_{\nu}^{2}\right)$. As discussed in Section 2, several UVIS occultation datasets show signatures of diffracted light while others appear to have no diffraction signals or possible detections of signals. These results appear to correlate with the ISS images of collisional activity or quiescence in the $\mathrm{F}$ ring at the time and longitude of the UVIS occultations. We use our models to assess potential correlations with the detection of UVIS diffraction signatures and physical properties of the $\mathrm{F}$ ring, such as the particle size distribution, the optical depth and the width of the ring.

Although we model the $\mathrm{F}$ ring assuming homogeneity across the width of the ring, we cannot assume azimuthal homogeneity of the F ring. During the course of the occultation (typically $20-60$ seconds), the ring particles will have moved substantially along their orbit. At an orbital velocity of approximately $20 \mathrm{~km} / \mathrm{s}$, the ring particles will be displaced by $400-1200 \mathrm{~km}$, which corresponds to anywhere between $0.023^{\circ}-0.17^{\circ}$, depending on the line of sight distance of Cassini to the rings at the time of the observation. The angular diameter of the Sun is $0.056^{\circ}$. Thus, a region of the $\mathrm{F}$ ring with a very different particle population could be occulting the Sun at the end of the solar occultation than was occulting the Sun at the beginning of the occultation. Therefore, in addition to modeling the entire occultation light curve, we also evaluate the best fitting models to the 'inner' and 'outer' edges of the ring individually. The split between the inner and outer edges was determined by calculating the midpoint in time of the occultation. The best-fit parameters for all three ring models (full ring, inner edge, and outer edge) are shown in Figures 12 - 14 for each occultation. This split is essentially modeling two independent regions of the ring (beginning and end of occultation); however, we use inner and outer ring edge as a reference to the location of the data and models along the light curve.

\subsection{Assessing the Results}

Because there is some degeneracy in results produced by varying $a_{\min }$ and $q$ (Becker et al., 2016), as well as in the results produced by variations of $W$ and $\tau_{\text {avg }}$, we also assess the best fitting models' effective particle size, $a_{\text {eff }}$ 
and equivalent depth, $W_{d}$, which combine these parameters. We calculate the effective particle size as presented in Cuzzi et al. (2009), following the equation

$$
a_{e f f}=\sqrt{\frac{\int_{a_{\min }}^{a_{\max }} a^{4} n(a) d a}{\int_{a_{\min }}^{a_{\max }} a^{2} n(a) d a}} .
$$

We define the equivalent depth as

$$
W_{d}=W \tau_{a v g}
$$

$W_{d}$ and $a_{\text {eff }}$ combine the four free parameters into two, and can be used to more easily evaluate the differences between the best fitting models of each occultation.

For each occultation, we evaluate the best fitting models in three categories: $a_{\min }=1 \mu \mathrm{m}, 1 \mu \mathrm{m}<a_{\min } \leq 10 \mu \mathrm{m}$, and $a_{\min } \geq 50 \mu \mathrm{m}$. In the first column of Figures 12 - 14, we show the best fitting model from each of these three categories. This illustrates where models with small, medium, or large $a_{\text {min }}$ fit or do not fit the data.

Our results show that models with a size distribution with $a_{\min }=1 \mu \mathrm{m}$ are often a good, if not the best fit to many of the occultations. Although a population of particles of this size has not been observed in previous observations, the short wavelengths of UVIS and the unique geometry of Cassini may enable the first detection of such a population. While these results could therefore have real implications for a persistent population of $1 \mu \mathrm{m}$ particles in the $\mathrm{F}$ ring, we note that models with this size distribution introduce another degeneracy in the results. When the majority of the particles are $1 \mu \mathrm{m}$, they diffract much of the light outside of the UVIS FOV. Therefore, a model with $a_{\min }=1 \mu \mathrm{m}$ and a relatively small equivalent depth can produce a similar light curve depth as a model with a large $a_{m i n}$ and larger equivalent depth, while also lacking strong diffraction 'ears' beyond the ring edges because the light has been completely diffracted out of the FOV. Thus, while the results that show $a_{\text {min }}=1 \mu \mathrm{m}$ models are the best fit may be real, it may also be a degeneracy in the model. Furthermore, we do not note any strong correlations with these results and the presence of a UVIS diffraction signal. Since our goal is to look for systematic changes in the F ring properties, we report the best fitting models for size distributions with both $a_{\text {min }}=1 \mu \mathrm{m}$ and $a_{\min }>1 \mu \mathrm{m}$ in Table 7 . 
In the second column of Figures 12 - 14, we show a contour plot of the goodness of fit of $W_{d}$ vs. $a_{e f f}$. In order to capture slight changes in $\chi_{\nu}^{2}$ while also plotting all results on the same contour color scheme, we plot $\left(\chi_{\nu}^{2}\right)$ on a logarithmic scale, with dark blue indicating regions of models with the best fits to the data.

We record the best fit parameters for models with $a_{\text {min }}=1 \mu \mathrm{m}$ and $a_{\text {min }}>$ $1 \mu \mathrm{m}$ in Table 7 . The table displays the best-fit model's $a_{m i n}, q, \tau_{\text {avg }}$, and $W$, as well as the resulting equivalent depth $\left(W_{d}\right)$ and effective particle size $\left(a_{e f f}\right)$, which we round to two significant figures.

We note here that the quantitative results from our models could be affected by neglecting limb darkening in our model. If the true angular disk of the Sun in the UV is smaller than that modeled here as a result of limb darkening, then our modeling efforts will favor larger ring widths than they would for a smaller disk. Additionally, the diffraction "ears" would be detected at distances farther from the (smaller) Sun than currently modeled, and therefore would indicate that a smaller effective particle size would likely be necessary to properly fit the data.

\subsubsection{Occultations with Diffraction Signatures}

Rev 43 (E): We begin with the Rev 43 (E) solar occultation since it was the clearest case of a properly-pointed solar occultation during which a diffraction signature was detected. In this data set, there were multiple data points that were $3 \sigma$ detections of light above the baseline, unocculted solar signal. Compared to some of the model fits to other occultations, however, the $\chi_{\nu}^{2}$ of the best fitting models for Rev 43 (E) are relatively high, even for the individual ring edges. Although the general shape of the light curve is well reproduced, including the depth of the occulted signal and the overall strength of the diffraction signal, the model still results in a relatively poor fit numerically. The $\chi_{\nu}^{2}$ is a reflection of the fit to each data point and is therefore sensitive to the fact that the magnitude of the signal at each time step in the light curve are offset. For example, in the outer ring edge model (bottom left figure for Rev 43, Fig. 12), the number of counts per second is predicted to be lower at each time step than is seen in the data. This is likely caused by our simplistic model of a constant optical depth across the F ring; as seen in the ISS images in Figure 2, the shape and optical depth of the ring are likely much more complex, especially in a region with a recent collisional event. Since the shape of the F ring light curve and the intensity of the diffraction signature are visibly good fits to the data, we trust the 
resulting implications for particle size distribution and equivalent depth of the ring despite the large values of $\chi_{\nu}^{2}$.

The Rev 43 (E) results show that for the outer F ring edge, a size distribution with a slope consistent with collisional activity $(q=3.2)$ and a small minimum particle size $\left(a_{\min }=2.5 \mu \mathrm{m}\right)$ fits the data best, assuming a high optical depth $(\tau=0.07)$ and a relatively thin F ring $(\mathrm{W}=350 \mathrm{~km})$. Despite the width, the resulting equivalent depth $\left(W_{d}=24.5\right)$ is the largest of any occultation, with the exception of the inner edge of Rev 43 (E). The effective particle size for this model is $219 \mu \mathrm{m}$.

The inner edge of the $\mathrm{F}$ ring is best modeled by either a steep particle size distribution with $a_{\min }=1 \mu \mathrm{m}, q=4.0$, or a moderately-sloped size distribution with $a_{\min }=100 \mu \mathrm{m}$ and $q=3.2$. As discussed in the beginning of this section, the $1 \mu \mathrm{m}$ model fits well because a significant amount of the signal is diffracted out of the FOV, resulting in a model with no diffraction ear and therefore fits the data for the inner edge of the F ring well. Focusing on the models with $a_{\min }>1 \mu \mathrm{m}$, we find that for the inner $\mathrm{F}$ ring, where no diffraction signature was detected, $a_{e f f}=430 \mu \mathrm{m}$, which is approximately twice as large as that of the outer $\mathrm{F}$ ring edge, where a diffraction signature was detected. We also find that the equivalent depth of the inner $\mathrm{F}$ ring is slightly larger than that of the outer $\mathrm{F}$ ring edge, and that both are very large compared to any other occultation event. This could be the result of the observed collision, causing a release of ring particles on the order of a few microns in size, that increase the optical depth of the ring in the UV.

Rev 9 (I): This occultation is complicated by the unknowns of the exact location of the Sun in the FOV and the variation in the unocculted solar signal; however, models with small minimum particle sizes do a relatively good job of reproducing the complicated shape of the Rev 9 (I) occultation. Since only a small portion of the Sun was visible in the FOV, it is likely that the full ring model best represents the $\mathrm{F}$ ring at the time of the occultation, although the best fitting models for each ring region have similar results. The best fitting model of the full ring reproduces the observed horns in the data, though the dip in the center of the occultation, when the ring is fully blocking the Sun, is not quite as deep as seen in the data. The effective particle size of the $\mathrm{F}$ ring is the same for the $1 \mu \mathrm{m}$ fit as the $2.5 \mu \mathrm{m}$ fit (193 microns). The equivalent depths $(7.5 \mathrm{~km})$ are generally consistent among all the best fitting models as well, but are the smallest of any of the solar occultation events.

Rev 55 (E): For the Rev 55 (E) occultation, the models fit the light curve 
relatively well, but do not completely capture the extent of the diffraction signal. Still, the best fitting models indicate the outer ring edge has an effective particle size of $219 \mu \mathrm{m}$ and an equivalent depth of $15 \mathrm{~km}$. The inner ring edge requires $a_{e f f}=410 \mu \mathrm{m}$ and $W_{d}=20.3 \mathrm{~km}$. Like for the Rev $43(\mathrm{E})$ models, the effective particle size of the inner edge of the ring (where no UVIS diffraction signature was detected) requires an effective particle size nearly twice the value required for the outer ring edge (where a UVIS diffraction signal was detected). Also similarly, the equivalent depth for the inner ring edge (edge with a diffraction signature) is slightly higher than the outer edge, which lacks a diffraction signature.

Rev $62(\mathbf{E})$ : This occultation is the most difficult to model, in part due to the few data points collected during the occultation because of Cassini's shorter line of sight distance to the rings. At the inner ring edge, the model fits well, closely reproducing the shape and signal magnitude of the data. The best fitting model indicates $a_{e f f}=510 \mu \mathrm{m}$ and $W_{d}=17.5 \mathrm{~km}$. The outer edge is not well reproduced by the model. Like with Rev 43 (E), this could be the result of our simplistic assumption of the optical depth; however, in this case, the best fitting model does not adequately replicate the depth of the $\mathrm{F}$ ring light curve or produce what would be a detectable signal. The best fitting model shows $a_{e f f}=740 \mu \mathrm{m}$ and $W_{d}=20.3 \mathrm{~km}$. Unlike for the other three models, these results indicate that the ring region that produces diffraction has a larger effective particle size and equivalent depth than the side of the ring that does not produce diffraction. However, the reduced $\chi_{\nu}^{2}$ of this fit is the worst of all ring edges, and so all the fit parameters are highly suspect.

Summary: For both Rev 43 (E) and Rev 55 (E), the effective particle size near the edge where a diffraction signature was detected was equal to 219 microns, while on the edge of the ring where a diffraction signature was not detected, the particle size was > 400 microns. In Rev 9 (I), for which diffraction is detected across the entire occultation, the effective particle size is 132 microns. Rev 62 (E) does not follow this trend; however, due to the poor fits, we do not have high confidence in the model results of this occultation. We note the emerging trend that the ring edges with UVIS diffraction signatures tend to be best reproduced by models with smaller effective particle sizes, and the regions without diffraction signatures have larger effective particle sizes. 


\subsubsection{Occultations without Diffraction Signatures}

Rev 59 (I): Our models appear to reproduce the F ring light curve during the Rev 59 (I) solar occultation very well (Figure 13). Despite being a relatively long occultation, a simple optical depth profile seems to capture signal from the entire light curve and the individual ring regions. The best fitting models with $a_{\min }>1 \mu \mathrm{m}$ show that the outer edge is best modeled using $a_{e f f}=710 \mu \mathrm{m}$ and $W_{d}=17.5 \mathrm{~km}$. The inner ring edge similarly suggests $W_{d}=17.5 \mathrm{~km}$, and $a_{e f f}=510 \mu \mathrm{m}$. The goodness of fit as well as the similar resulting properties of both ring regions suggest the $\mathrm{F}$ ring was in fact quiescent and somewhat uniform during this occultation.

We note here that the best fitting models for the outer edge indicate $a_{\text {min }}=1 \mu \mathrm{m}$, but that this model results in a very low effective particle size $\left(a_{e f f}=30 \mu \mathrm{m}\right)$, low optical depth $(\tau=0.02)$, and low equivalent depth $\left(W_{d}=10 \mathrm{~km}\right)$. Although we cannot rule this model out, the models with a slightly larger $a_{\text {min }}$ fit the data well and are more consistent with other results in this work and previous studies.

Rev 62 (I): The second solar occultation without any clear diffraction signatures is Rev $62(\mathrm{I})$. The best fitting models for $a_{\min }>1 \mu \mathrm{m}$ show that

$a_{e f f}=740 \mu \mathrm{m}$ and $W_{d}=15 \mathrm{~km}$ for the outer ring edge and $a_{e f f}=400 \mu \mathrm{m}$ and $W_{d}=11.6 \mathrm{~km}$ for the inner ring edge. Although the equivalent depth is slightly smaller than the Rev 59 (I) occultation, the effective particle sizes are similar.

Summary: The effective particle size for both occultations with no diffraction signatures ranges from $400-740$ microns, similar to the values detected for the non-diffraction signature edge of the $\mathrm{F}$ ring in the previous section. Both edges of both occultations have moderate to high equivalent depth values $(11.6-17.5 \mathrm{~km})$.

\subsubsection{Occultations with Possible Diffraction Signatures}

Rev 55 (I): There is a possible detection of a UVIS diffraction signature interior to the $\mathrm{F}$ ring core in Rev 55 (I). The best models produce a small diffraction signature that does not extend as far as the one apparent in the data. The best fitting model of the inner edge does suggest a small effective particle size of 160 microns and an equivalent depth of $10 \mathrm{~km}$, while the best fitting model for the outer ring edge (without a UVIS diffraction signature detection) shows a larger effective particle size $\left(a_{\text {eff }}=710 \mu \mathrm{m}\right)$ and a slightly larger equivalent depth of $15 \mathrm{~km}$.

Rev 65 (I): The best fitting model results show a similar pattern to Rev 
55 (I): $a_{e f f}=190 \mu \mathrm{m}$ and $W_{d}=10 \mathrm{~km}$ for the ring edge with a possible diffraction signature, while the models for the ring edge without a diffraction signature show $a_{e f f}=390 \mu \mathrm{m}$ and $W_{d}=11.6 \mathrm{~km}$. The best fitting models nearly capture the extent of the potential diffraction signature and reproduce the shape of the light curve fairly well.

Rev 65 (E): Although Rev 65 (E) was a close observation and therefore has fewer data points defining the occultation, the models replicate the light curve well, including the outer ring edge with a possible diffraction signature. The best results for the outer ring edge shows $a_{\text {eff }}=70 \mu \mathrm{m}$ and $W_{d}=10 \mathrm{~km}$. The results for the inner ring edge, where no potential diffraction signature was identified, show $a_{e f f}=740 \mu \mathrm{m}$ and $W_{d}=20.3 \mathrm{~km}$, again consistent with the observed correlation between smaller $a_{e f f}$ and diffraction signatures.

Rev 66 (I): This solar occultation shows a potential diffraction signature interior to the $\mathrm{F}$ ring based on only one $3 \sigma$ data point. The best fitting model to reproduce the $\mathrm{F}$ ring light curve of the inner ring edge has an $a_{e f f}=70 \mu \mathrm{m}$ and $W_{d}=10.5 \mathrm{~km}$. The outer ring edge results in $a_{e f f}=50 \mu \mathrm{m}$ and $W_{d}=$ $10 \mathrm{~km}$. While the ring edge with a diffraction signature is consistent with other findings, the very small effective particles side of the outer ring edge is inconsistent.

Rev 66 (E): Somewhat surprisingly, the best fitting models to the Rev 66 (E) data are the ones that do not break up the ring into regions. For consistency, we still report the results from the inner and outer ring edge. In this occultation, both ring edges have possible detections of a UVIS diffraction signature. For the outer ring edge, $a_{e f f}=330 \mu \mathrm{m}$ and $W_{d}=10.5 \mathrm{~km}$. The inner ring edge best fitting models find $a_{e f f}=710 \mu \mathrm{m}$ and $W_{d}=15 \mathrm{~km}$. If we use the emerging correlation pattern that ring edges with diffraction signatures are best reproduced using models with relatively small effective particle sizes, then the difference between ring edges in this occultation could suggest that the diffraction signature detection near the outer ring edge is real, while the inner ring edge diffraction signature is not.

Summary: These occultations show a possible detection of a diffraction signature on one edge of the $\mathrm{F}$ ring. The models have trouble capturing both the light curve during the occultation by the ring and the diffraction signal simultaneously. Still, with the exception of Rev 66 (I), the effective particle size of all of the ring edges without possible diffraction signatures was at least twice the effective particle size determined for the ring edge with a possible detection of a diffraction signature. This difference in effective particle size is also consistent with the difference between occultations with and without 
clear diffraction signatures; the effective particle size seems to be less than $\sim 300 \mu \mathrm{m}$ for the majority of diffraction detections or possible detections, while ring edges with no diffraction signature detections are best reproduced by models with $a_{\text {eff }}>400 \mu \mathrm{m}$.

\section{Summary}

In this work we have analyzed eleven solar occultations by Saturn's F ring observed by Cassini UVIS. We model the competing light curves produced by the removal of direct sunlight by ring particles through absorption, scattering and diffraction, and the addition of sunlight through the diffraction of the indirect solar signal into the instrument's field of view. In four of the eleven solar occultations, we detected additional light above the direct, unocculted solar signal just before and/or after the F ring occults the Sun, indicative of unambiguous diffracted light. In the case of the misaligned Rev 9 occultation, when most of the Sun was placed outside of the FOV, the added light due to diffraction stretches across the entire region of the $\mathrm{F}$ ring. The solar signal is diffracted by the sub-mm particles in Saturn's F ring during every occultation, but the detection of the diffraction signatures before or after the occultation requires a population of particles that can diffract light more broadly, such as particles on the order of 10-50 microns or smaller. Larger particles diffract more narrowly, essentially reducing the depth of the light curve produced by the $\mathrm{F}$ ring occultation. If diffraction in the $\mathrm{F}$ ring is not properly accounted for, the $\mathrm{F}$ ring will appear to have a lower optical depth than in reality.

Our spectral analysis revealed a flat spectrum with no variation in transmission or in the shape of the F ring light curve as a function of wavelength, indicating the ring particles are much larger than the UV wavelengths and therefore have a radius of at least $0.2 \mu \mathrm{m}$.

Through our analysis and modeling of solar occultations by the F ring, we find that in most of the observations there must be a region of small (sub$\mathrm{mm}$ ) particles that extends $\sim 500 \mathrm{~km}$ in radius, which is in agreement with the analysis by French et al. (2012). We find that the equivalent depth of the ring is typically between 7.5 and $25 \mathrm{~km}$ depending on the size distribution of the ring particles. This is larger than the equivalent depths determined by observations using longer wavelengths (Bosh et al., 2002). From our models, we find that if the effective particle size is small, then smaller equivalent depths are favored, whereas for larger effective particle sizes, a larger equiv- 

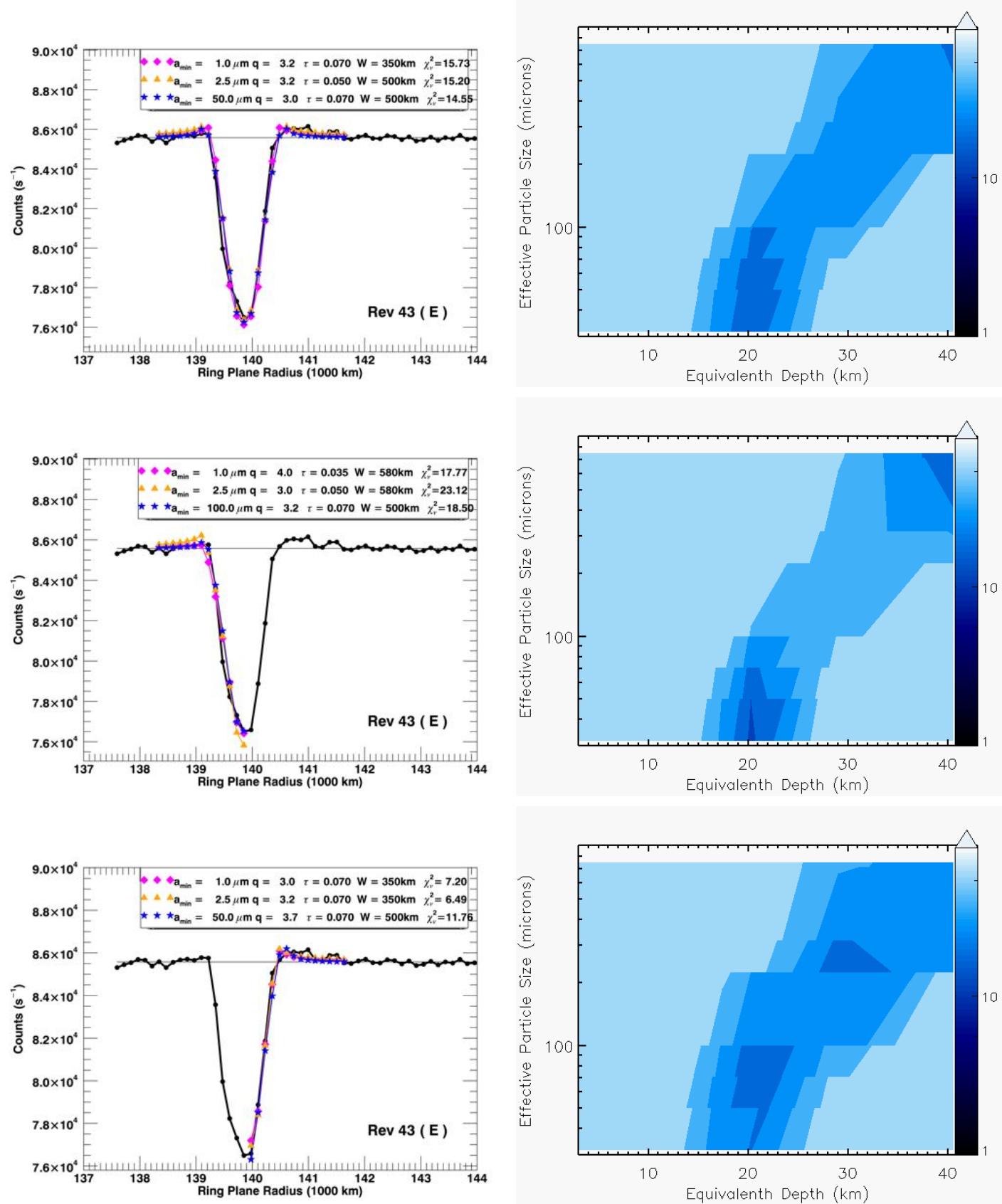

Figure 12: Model Results for UVIS Occultations with Diffraction Signatures: Rev 43 (E). The left column shows the data in black, and the best fit models for $a_{\min }=1 \mu \mathrm{m}$ (pink diamonds), $1<a_{m i n} \leq 10 \mu \mathrm{m}$ (orange triangles) and $a_{m i n}>10 \mu \mathrm{m}$ (blue stars). The full light curve model is shown on top, followed by the inner ring model in the middle, and the outer ring edge model at the bottom. The right column shows the contour plot for equivalent depth vs. effective particle size, dark blue contours representing the best models and light colors representing the worst. The contours are determined by taking the natural $\log$ of the $\chi_{\nu}^{2}$ results for each model. The color bar associated with the contour plot indicates the color as a function of $\chi_{\nu}^{2}$. 

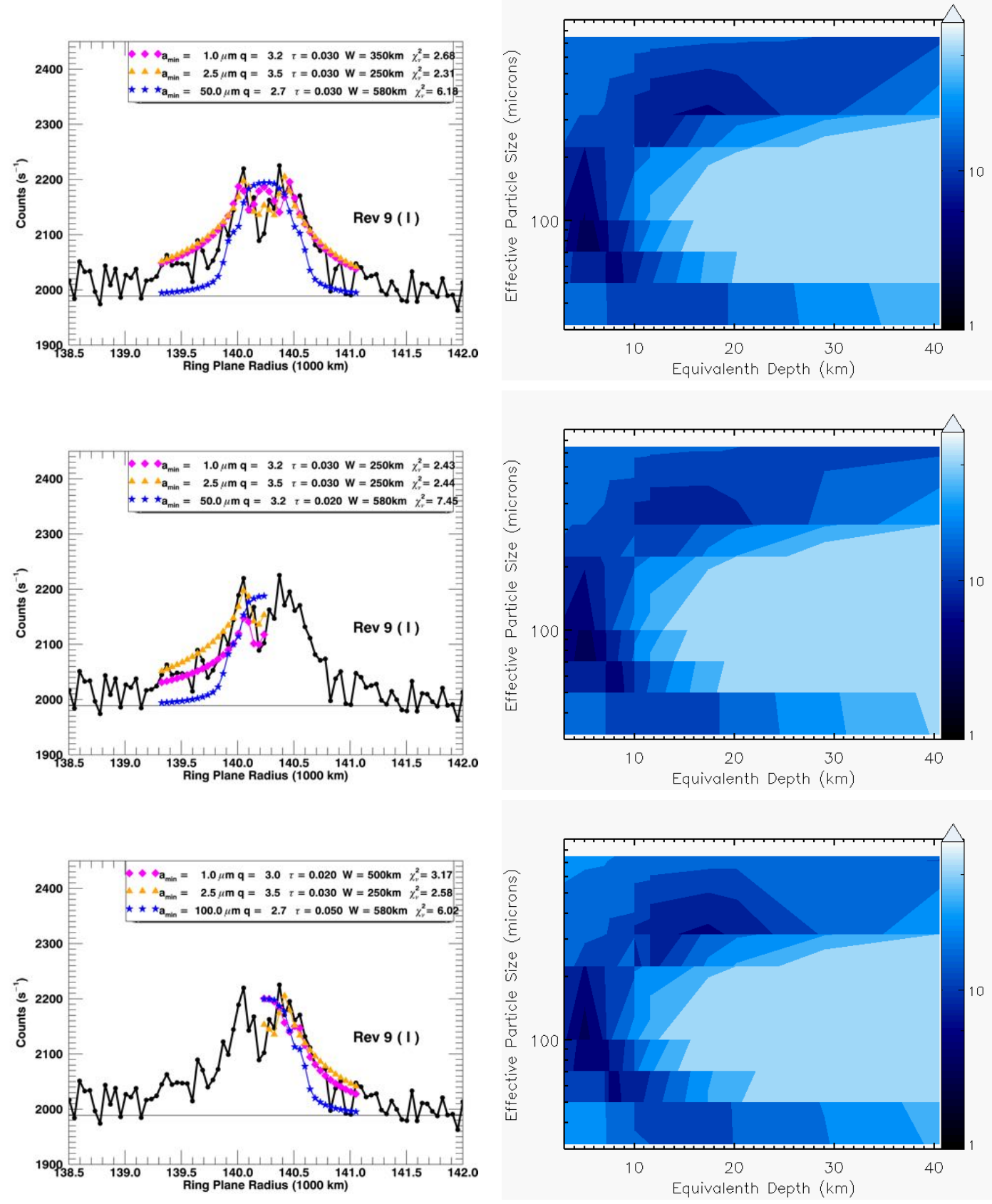

Figure 12 (cont.): Model Results for UVIS Occultations with Diffraction Signatures: Rev 9 (I) 

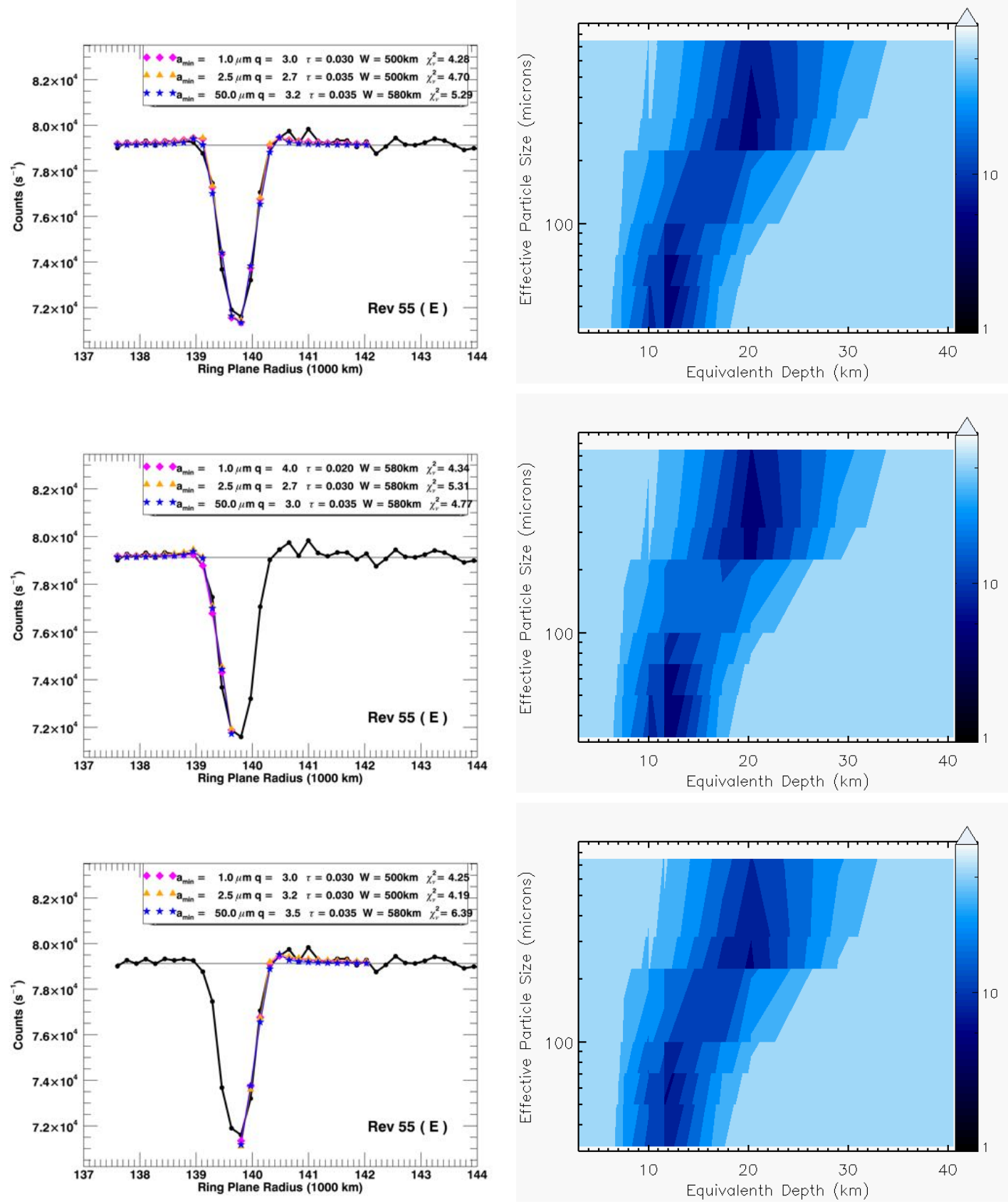

Figure 12 (cont.): Model Results for UVIS Occultations with Diffraction Signatures: Rev $55(\mathrm{E})$. 

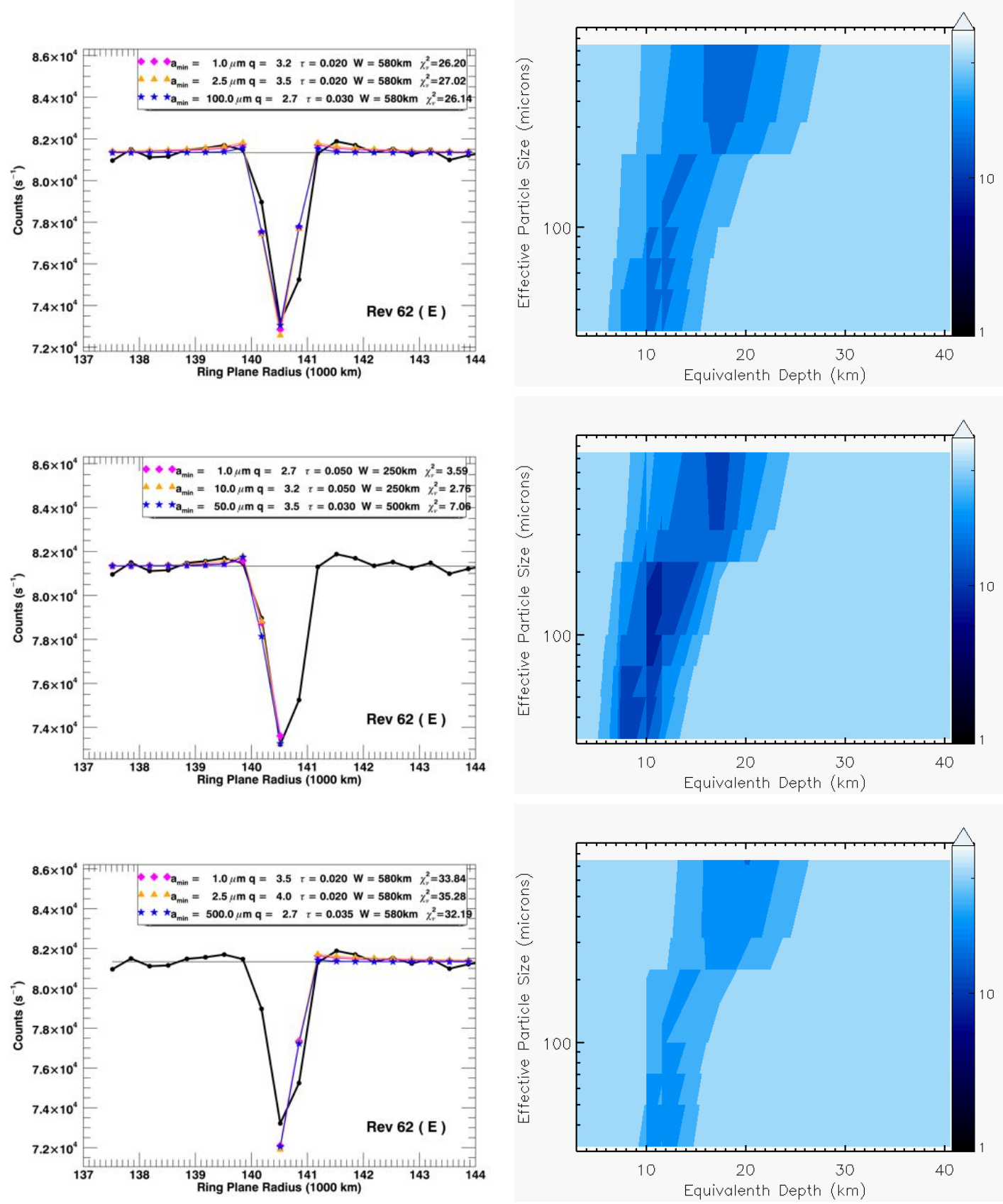

Figure 12 (cont.): Model Results for UVIS Occultations with Diffraction Signatures: Rev $62(\mathrm{E})$. 

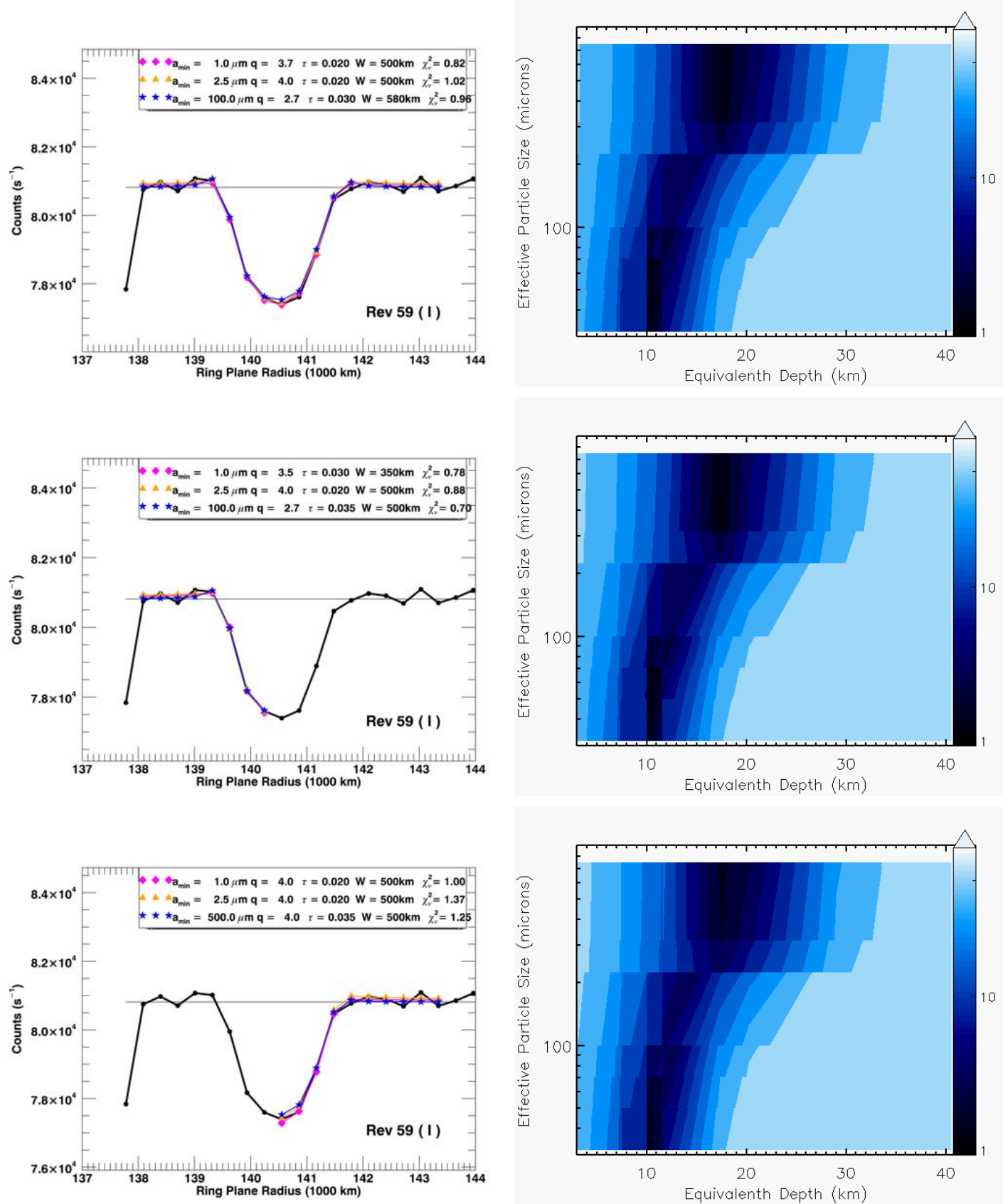

Figure 13: Model Results for UVIS Occultations with No Diffraction Signatures: Rev 59 (I). The first column shows the best fitting models to the light curves and the second column shows the contour plot for equivalent depth vs. effective particle size, as described in Figure 12. 

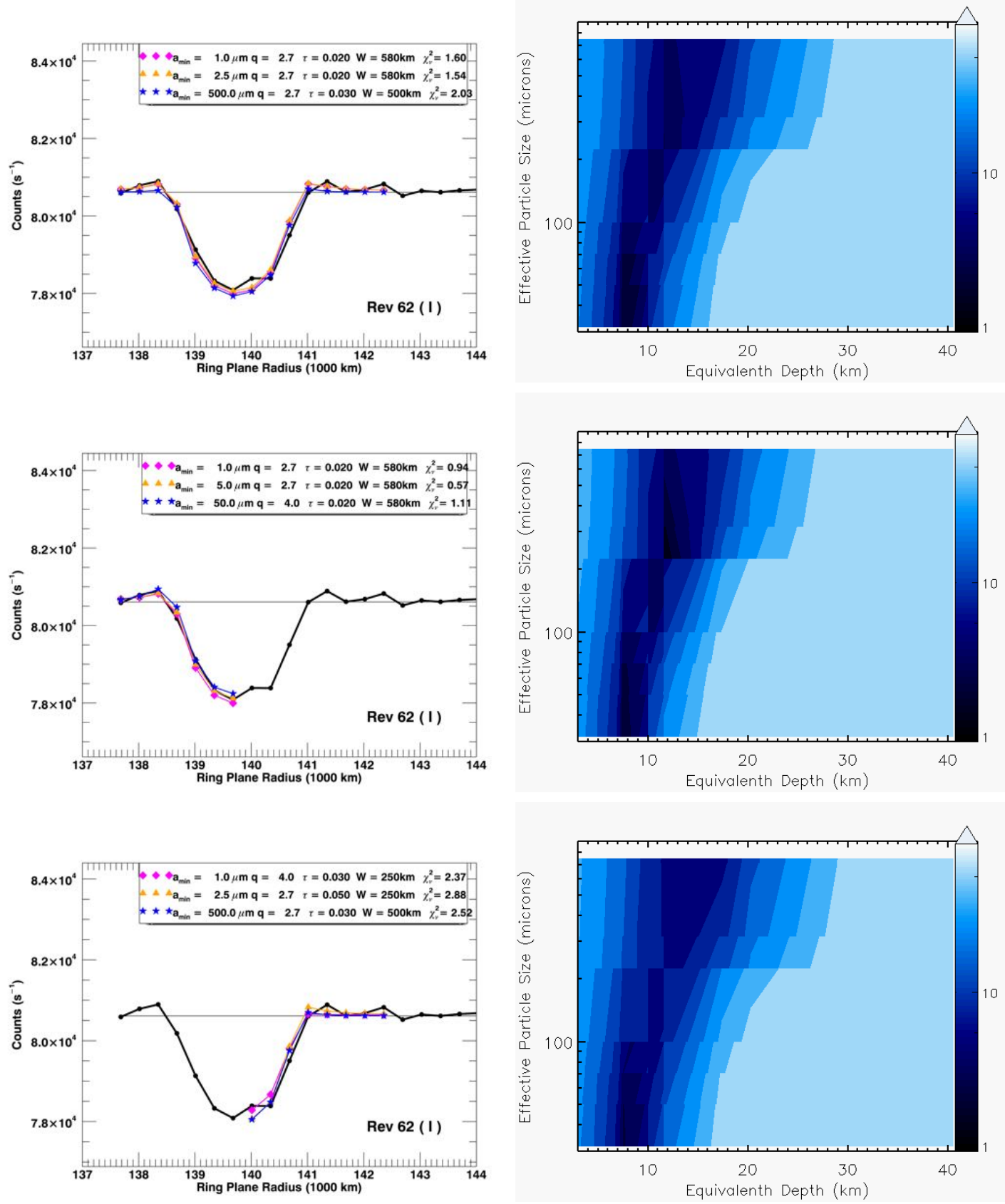

Figure 13 (cont.): Model Results for UVIS Occultations with No Diffraction Signatures: Rev 62 (I) 

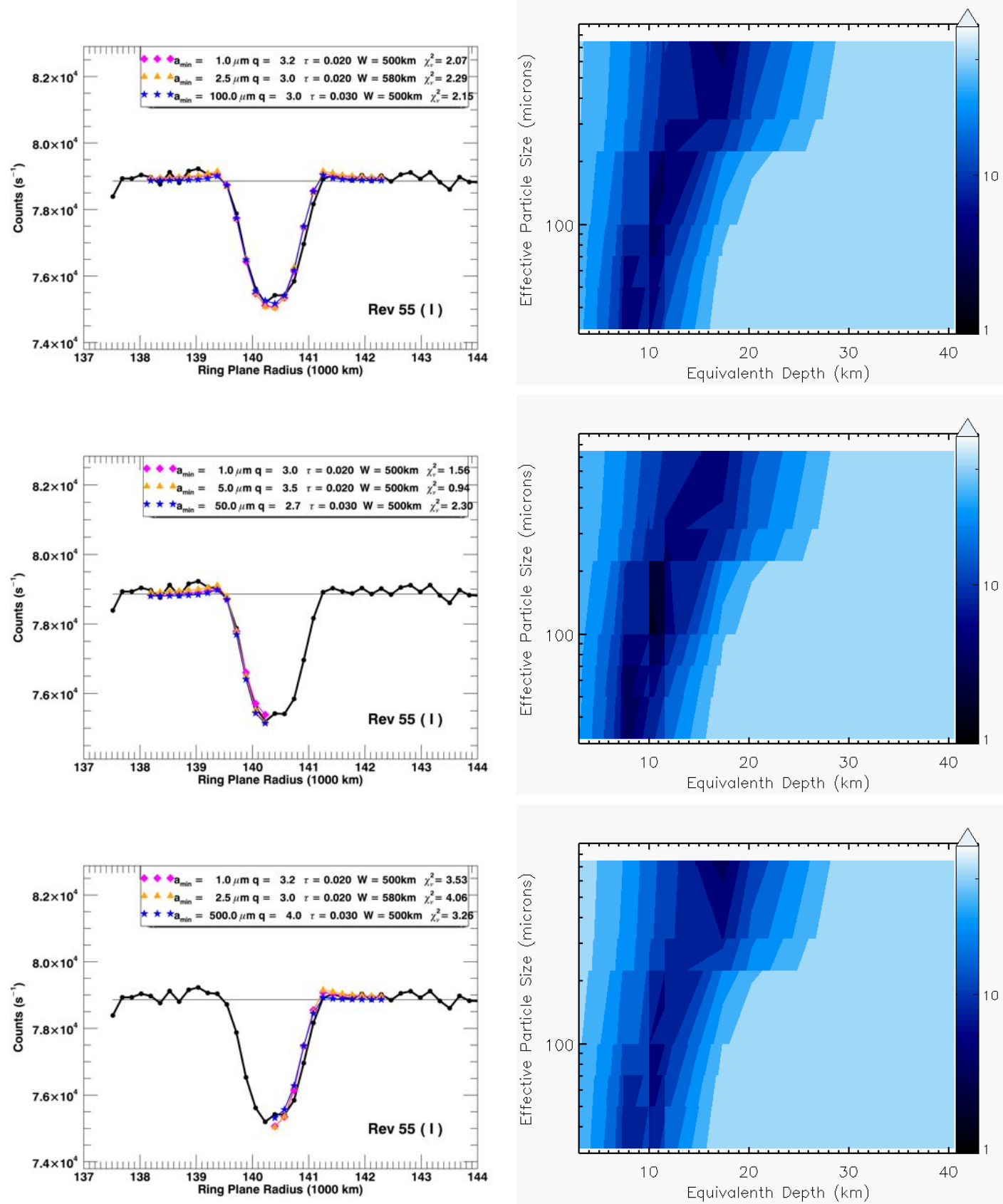

Figure 14: Model Results for UVIS Occultations with Possible Diffraction Signatures: Rev 55 (I). The first column shows the best fitting models to the light curves and the second column shows the contour plot for equivalent depth vs. effective particle size, as described in Figure 12. 

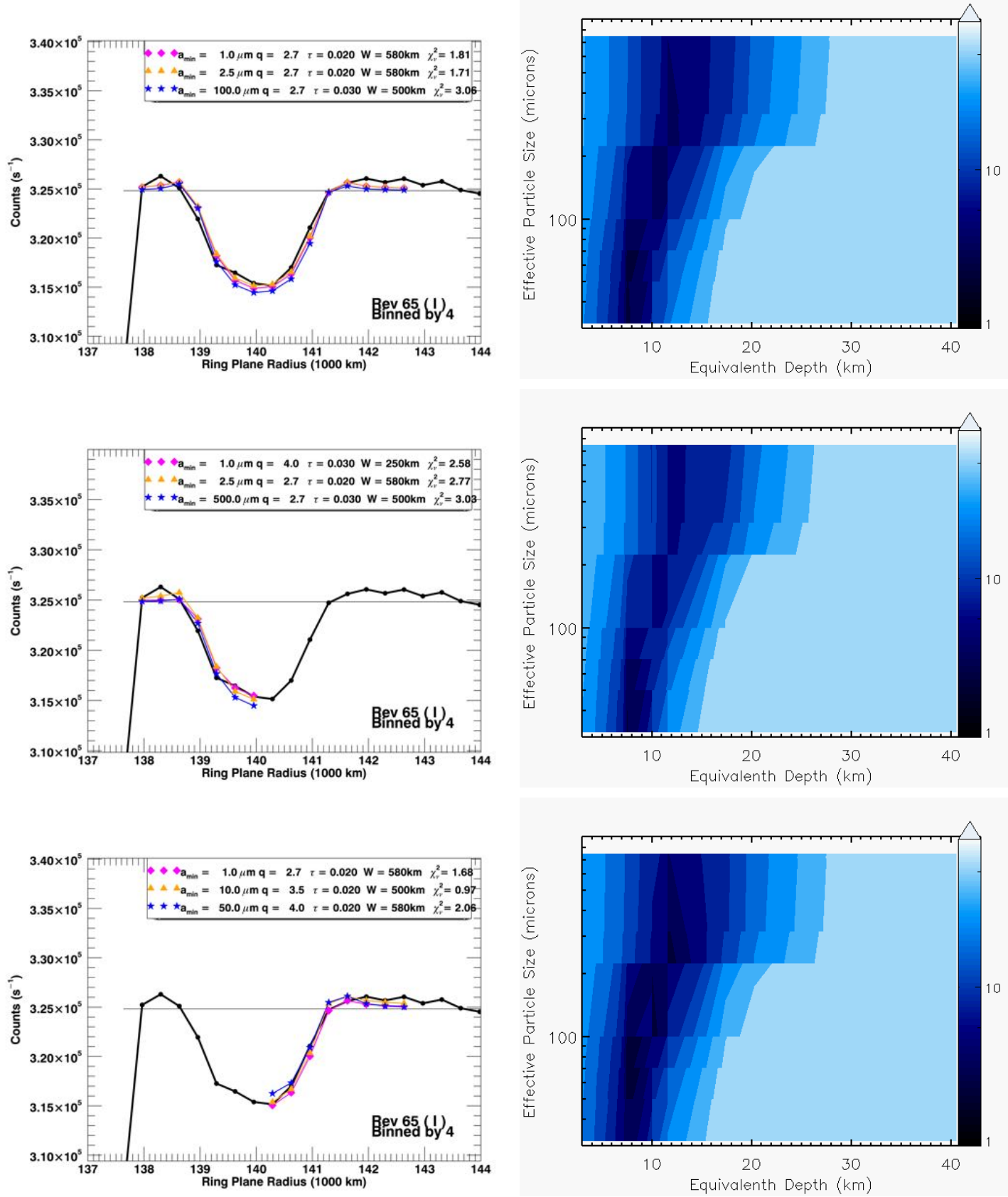

Figure 14 (cont.): Model Results for UVIS Occultations with Possible Diffraction Signatures: Rev 65 (I) 

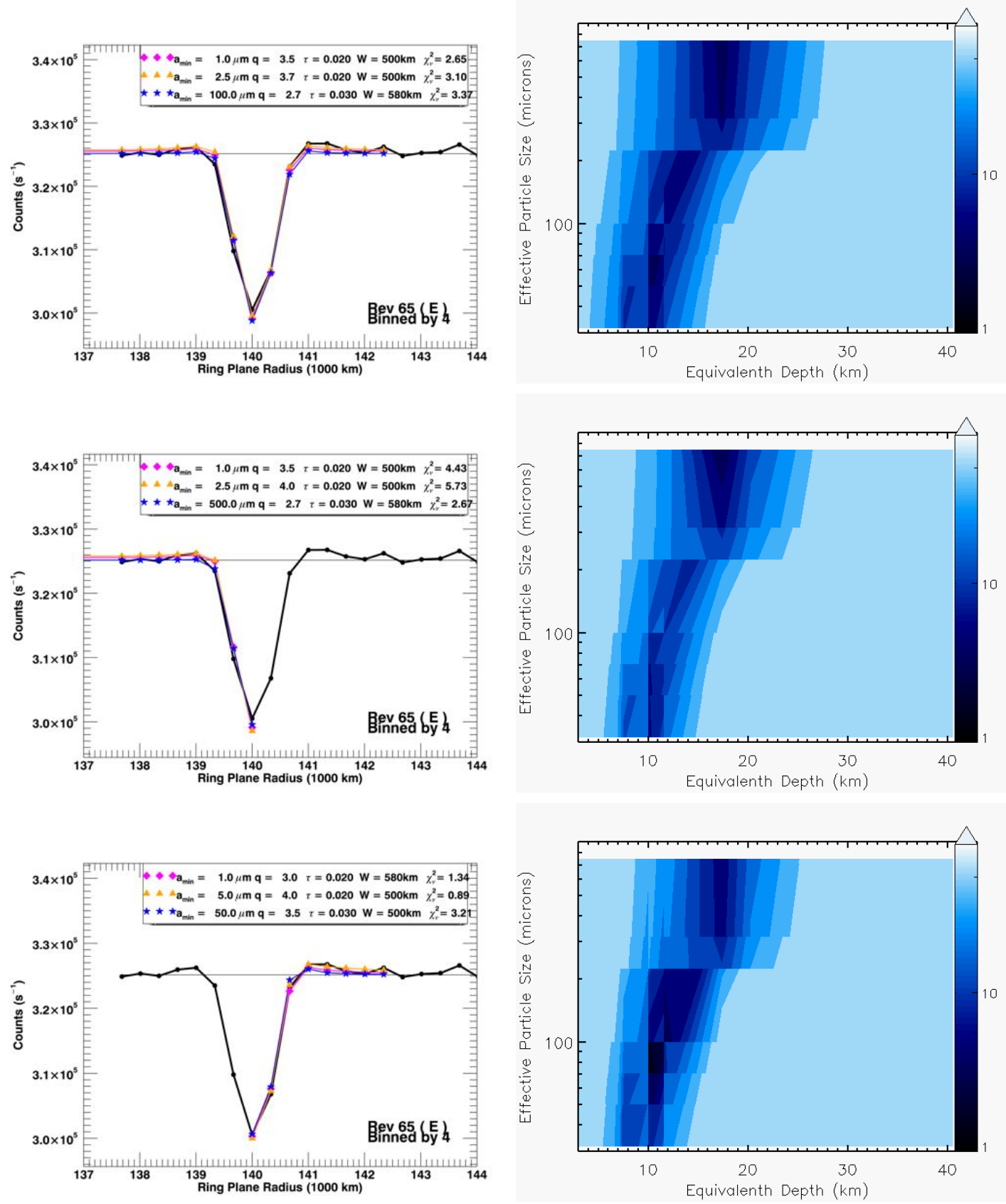

Figure 14 (cont.): Model Results for UVIS Occultations with Possible Diffraction Signatures: Rev $65(\mathrm{E})$ 

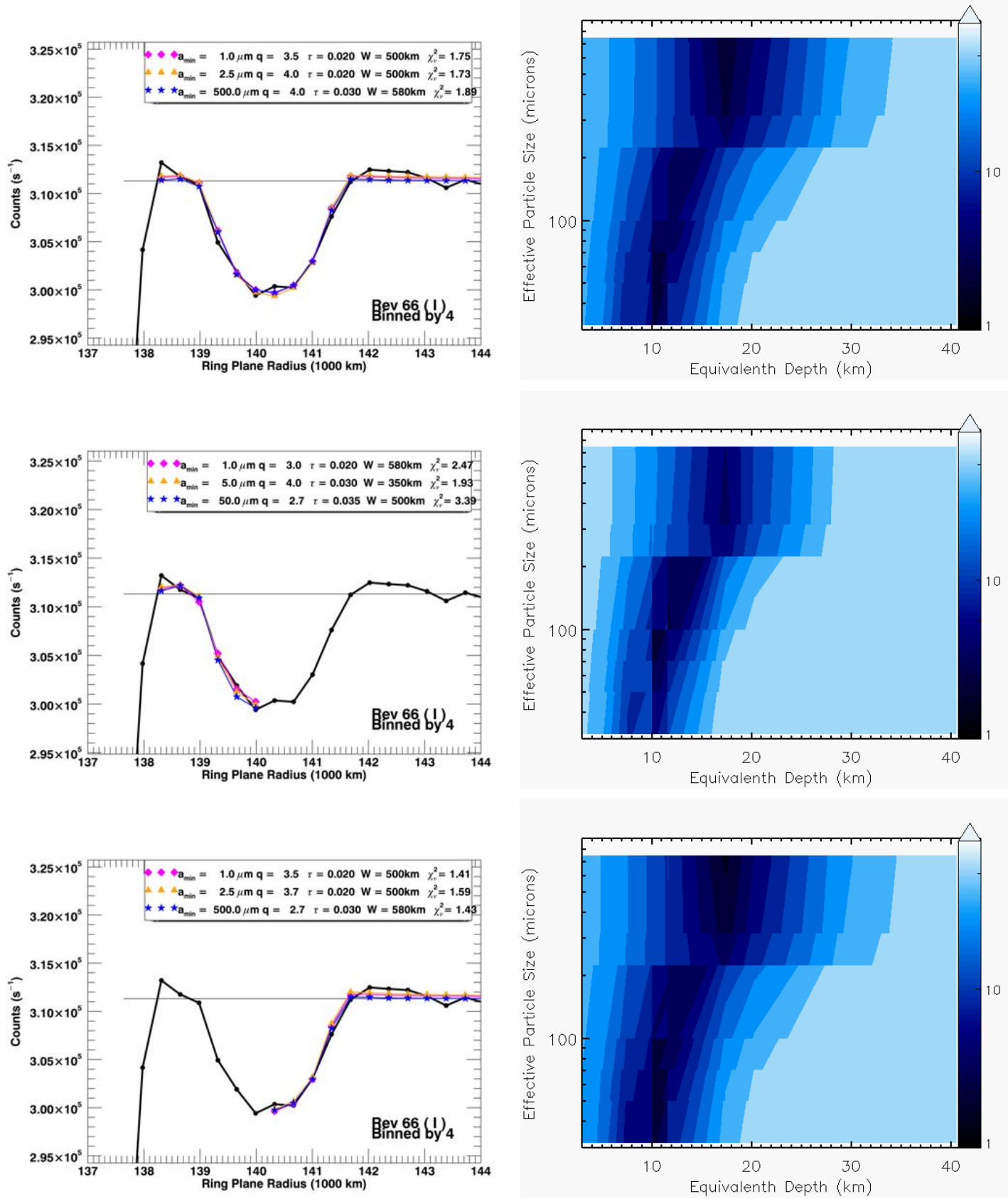

Figure 14 (cont.): Model Results for UVIS Occultations with Possible Diffraction Signatures: Rev 66 (I) 

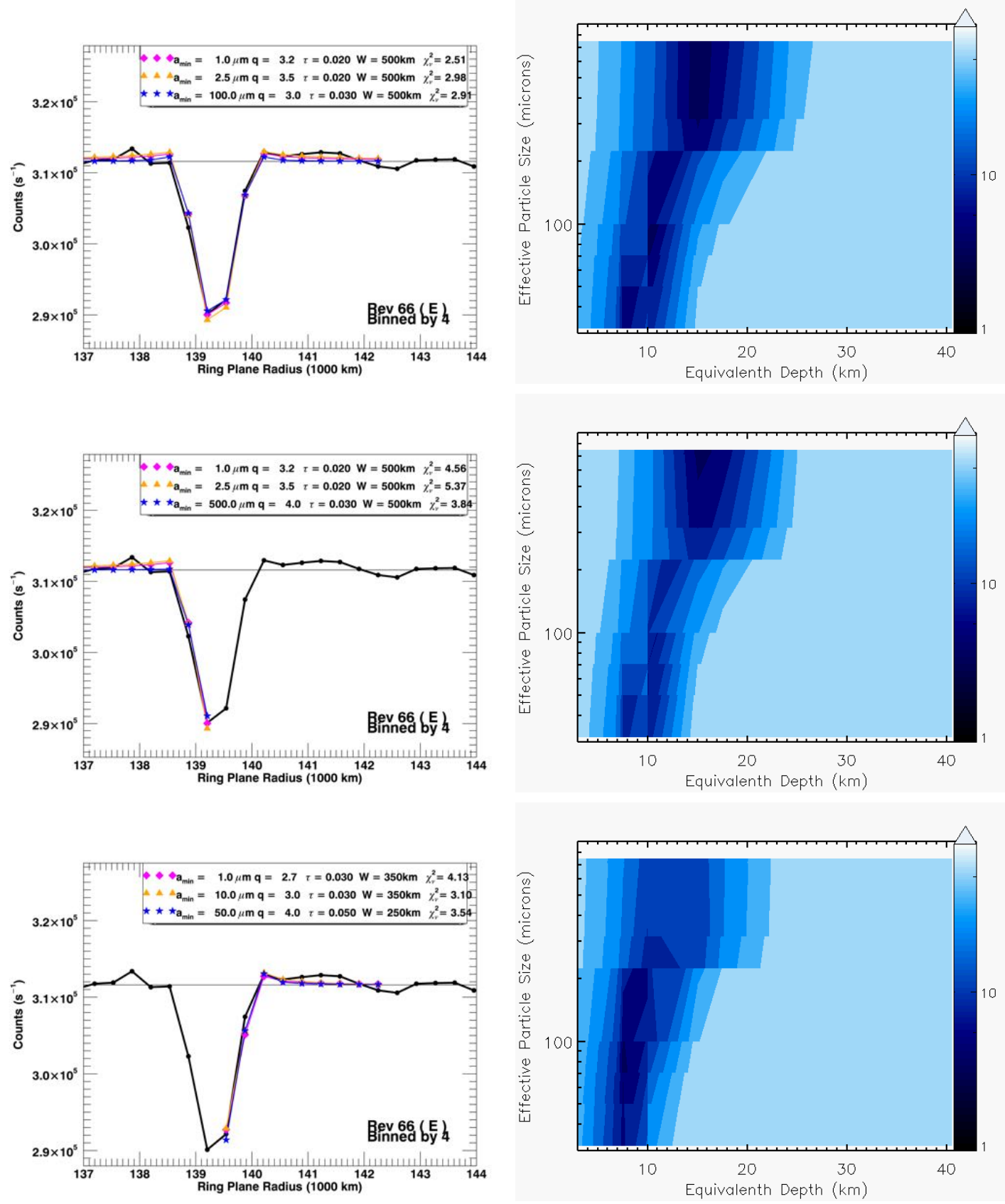

Figure 14 (cont.): Model Results for UVIS Occultations with Possible Diffraction Signatures: Rev $66(\mathrm{E})$ 
alent depth is preferred. The equivalent depth of the $\mathrm{F}$ ring during the Rev 43 (E) occultation is approximately twice the equivalent depth of most other occultations for any effective particle size. This appears to be due to the large collisional event described by (Murray et al., 2008) that occurred only months earlier in the same region of the ring.

Our modeling efforts generally show that the best fitting models to occultations where unambiguous diffraction signatures are detected require a minimum particle size $\leq 50$ microns, while occultations that do not show any additional signal above the direct, baseline solar signal require either a larger minimum particle size ( $\geq 100$ microns) or a shallow size distribution slope (small $q$ ), meaning the relative dominance of the smallest particles in the distribution is less. However, we do find some ambiguity in the models due to the multiple free parameters which include the average optical depth and width of the $\mathrm{F}$ ring, and the slope and minimum particle size of the size distribution. For example, a ring with a small $a_{\text {min }}$ and low optical depth $\tau_{\text {avg }}$ can produce a similar light curve to a ring with a larger $a_{\min }$ and a high value for $\tau_{\text {avg }}$. The models with larger $a_{\text {min }}$ values, however, cannot reproduce the observed diffraction signatures detected just before or after the occultation occurs.

Using the effective particle size $\left(a_{e f f}\right)$ instead, we find a trend that shows the $\mathrm{F}$ ring edges with a clear diffraction signature typically have $a_{\text {eff }}$ values of $<300$ microns, while edges without clear UVIS diffraction signatures are best reproduced by models with $a_{e f f}$ value of $>400$ microns. We also note a trend in which the ring edge with a diffraction signature usually has a slightly smaller equivalent depth than the edge without the diffraction signature in the same occultation. This may simply reflect variation in the ring, or may be related to a real change in either the optical depth or radial spread of the particles near and perhaps downstream from a collision.

When we compare our results for the UVIS occultations to ISS images of the same longitude in the $\mathrm{F}$ ring within a few weeks of the occultation, we find that the ISS images reveal signatures of a collisional event in the F ring core during all occultations in which an unambiguous diffraction signature is detected in the UVIS data. Similarly, the ISS images reveal a quiescent F ring at locations in which the UVIS occultation did not capture a diffraction signature. This suggests that the local variations detected in the UVIS data are reflective of ongoing activity in the $\mathrm{F}$ ring. The consistency between the ISS images, the UVIS occultation data, and the computational models suggest that the $\mathrm{F}$ ring envelope has a varying particle size distribution in 
which the smallest particles are replenished after observable collisional events occur in the F ring core. The strongest case for this scenario is the Rev 43 (E) solar occultation, during which one of the most prominent diffraction signals is observed in the UVIS data. The part of the ring that occulted the Sun during the Rev 43 (E) occultation was, serendipitously, the location of a very large disturbance in the $\mathrm{F}$ ring that was observed and described by (Murray et al., 2008) and produced a long-lasting brightening of the entire ring (French et al., 2012). The connection between the strongest diffraction signature in properly-pointed UVIS solar occultations and the occultation of one of the most notable large collisional events in the F ring strengthens the argument that the variations of the $\mathrm{F}$ ring described in this paper correspond to physical changes of the ring due to ongoing collisional events in Saturn's F ring.

Saturn's F ring is a dynamically-excited system. The particle size distribution of Saturn's F ring is a reflection of the on-going accretion processes countered by disruptive, collisional processes. Our analysis shows that it is not only the clearly-observed $\mathrm{F}$ ring core that is changing, but that the envelope of material that surrounds the ring is also continually evolving. UVIS solar occultations in which unambiguous diffraction signatures are observed appear to correspond with ISS images that show large collisional events in the ring, indicating an increase in the concentration of the smallest particles or an alteration of the particle size distribution through the release of very small particles that are short-lived in the ring envelope, or both. The size distribution of particles and the radial extent over which they are dispersed can be used to characterize the energy of the collisions that release them, which can constrain the origin and evolution of Saturn's complex F ring.

\section{Acknowledgements}

This material is based upon work supported by the National Aeronautics and Space Administration under grants NNX10AF20G and NNX15AH22G issued through the Cassini Data Analysis Program and through the Cassini Project on a grant issued through JPL to the UVIS investigation. This work was also supported by the NASA Florida Space Grant Consortium. C.M. was supported by the UK Science and Technology Facilities Council (Grant No. ST/M001202/1) and is grateful to them for financial assistance. N.A. is grateful to the UK Science and Technology Facilities Council for the award of a PhD studentship. The authors would like to thank Dr. Mark Showalter 
and Dr. Yanga Fernández for helpful discussions regarding this work. They would also like to thank the anonymous reviewers who significantly improved the content of this paper.

Albers, N., Sremčević, M., Colwell, J. E., Esposito, L. W., 2012. Saturn's F ring as seen by Cassini UVIS: Kinematics and statistics. Icarus 271, $367-$ 388 .

Amblard, P.-O., Moussaoui, S., Dudok de Wit, T., Aboudarham, J., Kretzschmar, M., Lilensten, J., Auchére, F., 2008. The euv sun as the superposition of elementary suns. Astronomy \& Astophysics 487, L13 - L16.

Attree, N. O., Murray, C. D., Williams, G. A., Cooper, N. J., 2014. A survey of low-velocity collisional features in Saturn's F ring. Icarus 227, 56-66.

Becker, T. M., 2016. Saturn's Rings: Measuring particle size distributions using Cassini UVIS occultation data. Ph.D. thesis, University of Central Florida.

Becker, T. M., Colwell, J. E., Esposito, L. W., Bratcher, A. D., 2016. Characterizing the particle size distribution of Saturn's A ring with Cassini UVIS occultation data. Icarus $279,20-35$.

Bosh, A. S., Olkin, C. B., French, R. G., Nicholson, P. D., 2002. Saturn's F ring: Kinematics and particle sizes from stellar occultation studies. Icarus $157,57-75$.

Bradley, T. E., Colwell, J. E., Esposito, L. W., Cuzzi, J. N.and Tollerud, H., Chambers, L., 2010. Far ultraviolet spectral properties of Saturn's rings from Cassini UVIS. Icarus 206, 458-466.

Burns, J. A., Showalter, M. R., Morfill, G. E., 1984. Planetary Rings. University of Arizona Press, Ch. The Etheral Rings of Jupiter and Saturn, pp. $200-272$.

Charnoz, S., Dones, L., Esposito, L. W., Estrada, P. R., Hedman, M. M., 2009. Saturn from Cassini-Huygens. Dordrecht:Springer, Ch. Origin and Evolution of Saturn's Ring System, p. 537.

Charnoz, S., Porco, C. C., Déau, E., Brahic, A., Spitale, J. N., Bacques, G., Baillie, K., 2005. Cassini discovers a kinematic sprial ring around Saturn. Science 310, 1300 - 1304. 
Colwell, J. E., Esposito, L. W., Jerousek, R. G., Sremčević, M., Pettis, D., Bradley, E. T., 2010. Cassini UVIS stellar occultation observations of Saturn's rings. The Astronomical Journal 140, 1569-1578.

Cooper, N. J., Murray, C. D., Williams, G. A., 2013. Local variability in the orbit of Saturn's F ring. The Astronomical Journal 145, $161-176$.

Cuzzi, J. N., 1985. Rings of Uranus: Not so thick, not so black. Icarus 63, 312-316.

Cuzzi, J. N., Burns, J. A., 1988. Charged particle depletion surround Saturn's F ring - evidence for a moonlet belt? Icarus 74, $284-324$.

Cuzzi, J. N., Clark, R. N., Filacchione, G., French, R. G., Marouf, E., Spilker, L., 2009. Saturn from Cassini-Huygens. Dordrecht:Springer, Ch. Ring Particle Composition and Size Distribution, p. 459.

Cuzzi, J. N., Pollack, J. B., 1978. Saturn's Rings: Particle composition and size distribution as constrained by microwave observations. Icarus 33, 233 -262 .

Dohnanyi, J. S., 1969. Collisional model of asteroids and their debris. Journal of Geophysical Research 74, 2531 - 2554.

Esposito, L. W., Barth, C. A., Colwell, J. E., Lawrence, G. M., McClintock, W. E., Stewart, A. I. F., Keller, H. U., Korth, A., Lauche, H., Festou, M. C., Lane, A. L., Hansen, C. J., Maki, J. N., West, R. A., Jahn, H., Reulke, R., Warlich, K., Shemansky, D. E., Yung, Y. L., 2004. The Cassini Ultraviolet Imaging Spectrograph Investigation. Space Science Reviews 115, 299-361.

Esposito, L. W., Meinke, B. K., Colwell, J. E., Nicholson, P. D., Hedman, M. M., 2008. Moonlets and clumps in Saturn's F ring. Icarus 194, $278-$ 289.

French, R. G., Nicholson, P. D., 2000. Saturn's Rings II: Particle sizes inferred from stellar occultation data. Icarus 145, 502-523.

French, R. G., Showalter, M. R., Sfair, R., Arüelles, C. A., Pajuelo, M., Becerra, P., Hedman, M. M., Nicholson, P. D., 2012. The brightening of Saturn's F ring. Icarus 219, 181 - 193. 
Grün, E., Morfill, G. E., Mendis, D. A., 1984. Plantary Rings. University of Arizona Press, Ch. Dust-Magentosphere Interactions, pp. 275 - 332.

Harbison, R. A., Nicholson, P. D., Hedman, M. M., 2013. The smallest particles in Saturn's A and C rings. Icarus 226, 1225-1240.

Hedman, M. M., Nicholson, P. D., Showalter, M. R., Brown, R. H., Buratti, B. J., Clark, R. N., 2011. The Christiansen Effect in Saturn's narrow dusty rings and the spectral identification of clumps in the $\mathrm{F}$ ring. Icarus 215, $695-711$.

Meinke, B. K., Esposito, L. W., Albers, N., Sremčević, M., 2012. Classification of $\mathrm{F}$ ring features observed in Cassini UVIS occultations. Icarus 218, $545-554$.

Murray, C. D., Beurle, K., Cooper, N. J., Evans, M. W., Williams, G. A., Charnoz, S., 2008. The determination of the structure of Saturn's F ring by nearby moonlets. Nature 453, $739-744$.

Murray, C. D., Chavez, C., Beurle, K., Cooper, N. J., Evans, M. W., Burns, J. A., Porco, C. C., 2005. How Prometheus creates structure in Saturn's F ring. Nature 437, Letters 1326 - 1329.

NAIF, 2015. Spice: An observation geometry system for planetary science missions. http://naif.jpl.nasa.gov/naif/.

Porco, C. C., Helfenstein, P., Thomas, P. C., Ingersoll, A. P., Wisdom, J., West, R. A., Neukum, G., Denk, T., Wagner, R., Roatsch, T., Kieffer, S., Turtle, E., McEwen, A., Johnson, T. V., Rathbun, J., Veverka, J., Wilson, D., Perry, J., Spitale, J. N., Brahic, A., Burns, J. A., Del Genio, A. D., Dones, L., Murray, C. D., Squyres, S., 2005. Cassini observes the active south pole of Enceladus. Science 311, 1393 - 1401.

Poulet, F., Sicardy, B., Dumas, C., Jorda, L., Tiphène, D., 2000. The crossings of Saturn ring plane by the Earth in 1995: Ring thickness. Icarus 145, $147-165$.

Showalter, M. R., Pollack, J. B., Ockert, E., Doyle, L. R., Dalton, B. J., 1992. A photometric study of Saturn's F ring. Icarus 100, $394-411$. 
Spitale, J. N., Jacobson, R. A., Porco, C. C., Owen Jr., W. M., 2006. The orbits of Saturn's small satellites derived from combined historic and Cassini Imaging Observations. The Astronomical Journal 132, $692-710$.

Tyler, G. L., Marouf, E. A., Simpson, R. A., Zebker, H. A., Eshleman, V. R., 1983. The microwave opacity of Saturn's rings at wavelengths of 3.6 and $13 \mathrm{~cm}$ from Voyager 1 radio occultation. Icarus 54, 160-188.

Vahidinia, S., Cuzzi, J. N., Hedman, M. M., Draine, B., Clark, R. N., Roush, T., Filacchione, G., Nicholson, P. D., Brown, R. H., Buratti, B. J., Sotin, C., 2011. Saturn's F ring grains: Aggregates made of crystalline water ice. Icarus $215,682-694$.

van de Hulst, H., 1957. Light Scattering by Small Particles. Dover Publications, Inc.

Zebker, H. A., A, M. E., Tyler, G. L., 1985. Saturn's rings: Particle size distributions for thin layer model. Icarus 64 (531-548). 
Table 2: ISS F Ring Mosaics

\begin{tabular}{|c|c|c|c|}
\hline $\begin{array}{l}\text { Associated UVIS } \\
\text { Solar Occultation }\end{array}$ & ISS Observation Name & $\begin{array}{c}\text { Start Time } \\
\text { (Year-DOY Time) }\end{array}$ & $\begin{array}{c}\text { End Time } \\
\text { (Year-DOY Time) }\end{array}$ \\
\hline $\operatorname{Rev} 9(\mathrm{I})$ & ISS_006RI_LPHRLFMOV001_PRIME & 2005-103 02:37:10.226 & 2005-103 16:22:52.908 \\
\hline Rev $43(\mathrm{E})$ & ISS_043RF_FMOVIE001_VIMS & 2007-108 02:36:35.536 & 2007-108 18:16:31.164 \\
\hline Rev 55 (I) & ISS_055RI_LPMRDFMOV001_PRIME & 2008-007 08:03:09.201 & 2008-007 22:59:18.822 \\
\hline Rev $55(\mathrm{E})$ & ISS_055RF_FMOVIE001_VIMS & 2007-365 15:47:29.178 & 2008-001 05:16:28.819 \\
\hline $\operatorname{Rev} 59$ (I) & ISS_059RF_FMOVIE002_VIMS & 2008-055 12:27:08.913 & 2008-056 04:21:11.493 \\
\hline Rev $62(\mathrm{I})$ & ISS_061RI_LPMRDFMOV001_PRIME & 2008-075 10:14:08.761 & 2008-076 00:19:38.402 \\
\hline Rev $62(\mathrm{E})$ & ISS_061RI_LPMRDFMOV001_PRIME & 2008-075 10:14:08.761 & 2008-076 00:19:38.402 \\
\hline $\operatorname{Rev} 65$ (I) & ISS_067RI_AZSCAN001_PRIME & 2008-131 06:32:20.126 & 2008-131 14:55:37.897 \\
\hline $\operatorname{Rev} 65(\mathrm{E})$ & ISS_067RI_AZSCAN001_PRIME & 2008-131 06:32:20.126 & 2008-131 14:55:37.897 \\
\hline $\operatorname{Rev} 66$ (I) & ISS_067RI_AZSCAN001_PRIME & 2008-131 06:32:20.126 & 2008-131 14:55:37.897 \\
\hline Rev $66(\mathrm{E})$ & ISS_067RI_AZSCAN001_PRIME & 2008-131 06:32:20.126 & 2008-131 14:55:37.897 \\
\hline
\end{tabular}

Details of the ISS mosaics that are compared with the UVIS solar occultations. The start and end times represent UTC start and end times for the ISS observations used to create the mosaic. 
Table 3: UVIS Diffraction Signatures and ISS Collision Features

\begin{tabular}{lcc}
\hline Occultation & UVIS Signature & ISS Collision Feature \\
\hline $\operatorname{Rev} 9(\mathrm{I})$ & yes & yes \\
$\operatorname{Rev} 43(\mathrm{E})$ & yes & yes \\
$\operatorname{Rev} 55(\mathrm{E})$ & yes & yes \\
$\operatorname{Rev} 62(\mathrm{E})$ & yes & yes \\
$\operatorname{Rev} 59(\mathrm{I})$ & no & no \\
$\operatorname{Rev} 62(\mathrm{I})$ & no & no \\
$\operatorname{Rev} 55(\mathrm{I})$ & possible & old collision \\
$\operatorname{Rev} 65(\mathrm{I})$ & possible & no \\
$\operatorname{Rev} 65(\mathrm{E})$ & possible & possible \\
$\operatorname{Rev} 66(\mathrm{I})$ & possible & no \\
$\operatorname{Rev} 66(\mathrm{E})$ & possible & possible \\
\hline
\end{tabular}

For each solar occultation we indicate whether a diffraction signature was detected, not detected, or possibly detected in the UVIS data and whether or not the corresponding ISS mosaics indicate a recent collisional event near the longitude of the $\mathrm{F}$ ring region that occulted the Sun in the UVIS data. Note that clear detections of diffracted light in the UVIS data correspond with clear collisional events in the ISS images, while clear non-detections in the UVIS data correspond with quiescent ring regions in the ISS images. 
Table 4: Slope Parameters for Spectral

\begin{tabular}{ccccccccc}
\hline Occultation & $\mathrm{A}$ & $\sigma_{A}$ & $\mathrm{~B}$ & $\sigma_{B}$ & $\mathrm{~A}$ (diffracted) & $\sigma_{\text {Adiff }}$ & $\mathrm{B}$ (diffracted) & $\sigma_{\text {Bdiff }}$ \\
$\operatorname{Rev} 9(\mathrm{I})$ & -0.123 & 0.164 & 0.0010 & 0.0019 & -0.166 & 0.037 & 0.0024 & 0.0004 \\
$\operatorname{Rev} 43(\mathrm{I})$ & -0.0953 & 0.0128 & -0.000106 & 0.00014 & -0.0059 & 0.0064 & 0.00013 & $7.22 \mathrm{E}-5$ \\
$\operatorname{Rev} 55(\mathrm{I})$ & -0.011 & 0.015 & -0.00044 & 0.00017 & -0.029 & 0.0123 & 0.00039 & 0.00014 \\
$\operatorname{Rev} 55(\mathrm{E})$ & -0.099 & 0.0138 & $4.96 \mathrm{E}-05$ & 0.00016 & -0.014 & 0.0098 & 0.00024 & 0.00011 \\
$\operatorname{Rev} 59(\mathrm{I})$ & -0.0051 & 0.017 & -0.00044 & 0.00019 & N/A & N/A & N/A & N/A \\
$\operatorname{Rev} 62(\mathrm{I})$ & -0.0708 & 0.0136 & 0.00043 & 0.00015 & N/A & N/A & N/A & N/A \\
$\operatorname{Rev} 62(\mathrm{E})$ & -0.0977 & 0.0163 & $-7.93 \mathrm{E}-06$ & 0.00018 & -0.0079 & 0.0101 & 0.000127 & 0.000114 \\
$\operatorname{Rev} 65(\mathrm{I})$ & -0.0669 & 0.0212 & 0.00047 & 0.00024 & -0.00167 & 0.00794 & $4.24 \mathrm{E}-05$ & $8.92 \mathrm{E}-05$ \\
$\operatorname{Rev} 65(\mathrm{E})$ & -0.0752 & 0.01488 & $-1.92 \mathrm{E}-05$ & 0.000167 & N/A & N/A & N/A & N/A \\
$\operatorname{Rev} 66(\mathrm{I})$ & -0.0519 & 0.01462 & 0.00021 & 0.00016 & N/A & N/A & N/A & N/A \\
$\operatorname{Rev} 66(\mathrm{E})$ & -0.0153 & 0.0162 & -0.00066 & 0.00018 & 0.0056 & 0.0083 & $-4.69 \mathrm{E}-05$ & $9.36 \mathrm{E}-05$ \\
\hline
\end{tabular}

The best-fit parameters in the linear equation $y=A+B x$ for each occultation, where $x$ is the wavelength in nm and $y$ is the value $\left(I_{\text {core }}-I_{0}\right) / I_{0}$, as shown in Figs 4 and 5. $\sigma_{A}$ and $\sigma_{B}$ represent the 1- $\sigma$ uncertainties on parameters $A$ and $B$, respectively. We apply the same linear fit where $y$ is the value $\left(I_{\text {diffraction }}-I_{0}\right) / I_{0}$ to find the best-fit parameters $\mathrm{A}$ (diffracted), B (diffracted), and their uncertainties $\sigma_{\text {Adiff }}$ and $\sigma_{\text {Bdiff }}$.

Table 5: F Ring Orbital Parameters

\begin{tabular}{ccccccc}
\hline$a_{s m}(\mathrm{~km})$ & $e$ & $i(\mathrm{deg})$ & $\Omega(\mathrm{deg})$ & $\tilde{\omega}(\mathrm{deg})$ & $\dot{\Omega}\left(\frac{\text { deg }}{\text { day }}\right)$ & $\dot{\tilde{\omega}}\left(\frac{\text { deg }}{\text { day }}\right)$ \\
\hline 140221.3 & $2.35 \times 10^{-3}$ & $6.43 \times 10^{-3}$ & 15.0 & 24.2 & -2.6877 & 2.7025 \\
\hline
\end{tabular}

Values listed are from (Albers et al., 2012) for Epoch J2000. $a_{s m}$ is the semi-major axis, $e$ is the eccentricity, $i$ is the inclination, $\Omega$ is the longitude of the ascending node, $\tilde{\omega}$ is the longitude of pericenter, $\dot{\Omega}$ is the regression rate, and $\dot{\tilde{\omega}}$ is the procession rate of the F ring. The longitudes are measured prograde from the ascending node of Saturn's equatorial plane on Earth's J2000 equator. 
Table 6: Variable Parameter Space

\begin{tabular}{cccc}
\hline $\mathrm{W}(\mathrm{km})$ & $\tau_{\text {avg }}$ & $a_{\min }(\mu \mathrm{m})$ & $q$ \\
\hline 100 & $0.03,0.05,0.07$ & $1,2.5,5,10,50,100,500$ & $2.7,3.0,3.2,3.5,3.7,4.0$ \\
250 & $0.03,0.05,0.07$ & $1,2.5,5,10,50,100,500$ & $2.7,3.0,3.2,3.5,3.7,4.0$ \\
350 & $0.03,0.05,0.07$ & $1,2.5,5,10,50,100,500$ & $2.7,3.0,3.2,3.5,3.7,4.0$ \\
500 & $0.02,0.03,0.035,0.05,0.07$ & $1,2.5,5,10,50,100,500$ & $2.7,3.0,3.2,3.5,3.7,4.0$ \\
580 & $0.02,0.03,0.035,0.05,0.07$ & $1,2.5,5,10,50,100,500$ & $2.7,3.0,3.2,3.5,3.7,4.0$ \\
\hline
\end{tabular}

Parameters used for each suite of models. $W$ is the F ring width, $\tau_{\text {avg }}$ is the average optical depth of the ring, $a_{\min }$ is the minimum particle size and $q$ is the slope of the particle size distribution. Note that all parameters are the same for each model, with the exception of the additional two $\tau_{\text {avg }}$ values for models with $W=500$ and $580 \mathrm{~km}$. 
Table 7: Best-fit Model Parameters

\begin{tabular}{|c|c|c|c|c|c|c|c|c|c|c|c|c|c|c|c|c|c|}
\hline \multirow{2}{*}{ Oevitates } & \multirow{2}{*}{ Eing argien } & \multicolumn{2}{|c|}{$\begin{array}{l}\text { Mnimum } \\
\text { sartide diet }\end{array}$} & \multicolumn{2}{|r|}{ a } & \multicolumn{2}{|r|}{ then } & \multicolumn{2}{|c|}{$\begin{array}{l}\text { Ene Width } \\
\text { nemb }\end{array}$} & \multicolumn{2}{|c|}{$\begin{array}{l}\text { Ehective Particle } \\
\text { Slise (micosil) }\end{array}$} & \multicolumn{2}{|c|}{$\begin{array}{c}\text { Equhalemi Cenoth } \\
\text { Dand }\end{array}$} & \multicolumn{2}{|c|}{$x_{v}^{2}$} & \multirow{2}{*}{$\begin{array}{l}\text { Evtraction } \\
\text { Sequare }\end{array}$} & \multirow{2}{*}{$\begin{array}{l}\text { Collivien } \\
\text { Stpruture }\end{array}$} \\
\hline & & 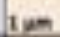 & $.1 \mathrm{~m}$ & $1 \mathrm{em}$ & b.1. & $1=$ & $b 1=n$ & 10 & onem & in & $.1 \mathrm{ym}$ & $1 y=$ & pin & $1 \mathrm{n}$ & ol in & & \\
\hline \multirow[t]{2}{*}{ Rev $43(0)$} & inner Ning Lefge & i & 100 & 40 & 12 & ebos & $a \geq ?$ & 30 & $\%$ & 10 & 69 & 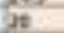 & is & $13 \pi$ & ins & 42 & \multirow[t]{2}{*}{$\mathrm{Ba}$} \\
\hline & aber Riv Ely" & 1 & 25 & 10 & 12 & $G D$ & a.s. & 160 & 180 & 190 & 220 & 245 & 205 & 3.2 & C.4B & Nen & \\
\hline \multirow{2}{*}{ Rev 900} & Solvingt & 1 & 25 & $\sqrt{13}$ & 1.5 & 90 & tas & 160 & J5a & 100 & 10 & iss & 75 & 20 & 213 & ris & \multirow[b]{2}{*}{ set } \\
\hline & near kine toge & 1 & 25 & 92 & 35 & 9.90 & 693 & J5e & 250 & 180 & 189 & is & 25 & 20 & 2.44 & $m$ & \\
\hline \multirow{3}{*}{ Berss [t] } & Avenive & i & 75 & 10 & 27 & 1000 & hass & 300 & 500 & 200 & 200 & 15 & 123 & 430 & 67 & 70 & \multirow{3}{*}{5 en } \\
\hline & mer Nine Idye & it & $\infty$ & 40 & 10 & laseg. & basm & 50 & 150 & 10 & 6)3 & 115 & 207 & 44 & $6 n$ & 43 & \\
\hline & aver kive fide" & 1 & 25 & 10 & 13 & G99 & 6.s. & 20 & $\operatorname{tg}$ & ins & 20 & 15 & is & 45 & eis & Tess & \\
\hline \multirow{3}{*}{ Rev 62 (5) } & fol Bing' & it & 100 & 32 & 27 & 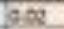 & bas & 300 & 80 & 150 & 523 & 119 & 174 & 262 & 280 & 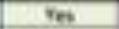 & \multirow{3}{*}{$5 \mathbf{m}$} \\
\hline & 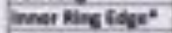 & $i$ & 10 & 27 & in & $\cos$ & has & Jue & na & ine & 20 & i2: & ins & 190 & 24 & 42 & \\
\hline & aen king fidye & it & $8 x$ & is & 33 & $9=0$ & a.sas & Ins & $\mathrm{seg}$ & tos & 169 & 116 & 209 & IEM & $n=$ & $m$ & \\
\hline \multirow{3}{*}{ hers9 (1) } & forping & 1 & 100 & 37 & 27 & $90 x$ & 63 & 20 & 50 & 10 & 573 & in & 1774 & lon & 394 & S? & \multirow{3}{*}{ Ne } \\
\hline & preet Ning tive" & 1 & 100 & 35 & 27 & 900 & bass & 190 & 500 & 100 & 539 & 103. & 1273 & 0.7 & 175 & s) & \\
\hline & 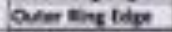 & 1 & 300 & 49 & 40 & a.00 & b.25s & 300 & 500 & 10. & 233 & 18. & 175 & 1 & 125 & 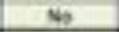 & \\
\hline คอง $62(1)$ & aver ting Idps & 1 & 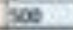 & 40 & 23 & Q600 & bas & 200 & 200 & 10 & 240 & 35 & is & 2.07 & 258 & ws & Ne \\
\hline & Sol Wist & 7 & $1 x$ & 32 & 20 & $9=9$ & lasa & 100 & $\infty$ & 10 & eis & 78 & is & कर & 125 & Rackb- & \\
\hline Aev 55 (1) & inner king Idye" & it & 5 & 10 & 15 & 192 & hat & 90 & 60 & 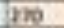 & 1360 & is & 10 & 146 & bs & Rosubie & Oid collitive \\
\hline & aper king fide" & 1 & $x$ & 32 & 60 & las & han & $\infty$ & $\infty$ & 180 & 32 & 18 & is & ass & bas & So & \\
\hline & foldinger & 1 & 25 & 27 & 25 & 0,02 & 6.22 & 300 & 880 & 180 & 200 & 118 & 146 & 181 & 171 & hasione & \\
\hline Bev 65 (1) & mertingtoge & 1 & 25 & 10 & 27 & 600 & bm & 750 & 50 & 10 & 40 & 73 & 1114 & a. & $2 \pi$ & N & ne \\
\hline & over by boge" & 1 & 10 & 27 & 35 & 100 & $\operatorname{lom}$ & 200 & 200 & 190 & 19 & 115 & 10 & $18 \pi$ & $2 n$ & nestes & \\
\hline & Ne tivg & 1 & 25 & 15 & 13 & $a \equiv$ & 222 & 500 & 90 & 100 & 9 & 16 & 10 & 205 & DI & 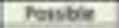 & \\
\hline Rer 65 (t) & ineer Ming The" & i & 800 & 25 & 27 & $a 00$ & bass & 300 & 290 & 100 & 340 & 11.5 & 203 & 4.43 & 257 & 40 & Nontaste \\
\hline & Ouper Bing Idpe" & $i$ & 5 & 10 & 10 & 9.09 & ha.2. & 500 & 500 & 10 & 20 & 11.6 & 10 & 14 & 20 & Powbie & \\
\hline & Fof kisc' & 1 & 25 & 35 & eb & 9.9 & a.2 & $3 \infty$ & 40 & 100 & 5 & 16 & 10 & $1 \times$ & 53 & Rotsble & \\
\hline Rev 6601 & ment Ring tidye" & t & 3 & 10 & 10 & 938 & 6,3 & 300 & 190 & too & 18 & 118 & ins & XeT & 193 & Facibe & No \\
\hline & aver bist talp & it & 25 & (3) & ED & $a=9$ & last & 100 & $\infty$ & $n$ & lse & 18 & te & 1 at & :43 & s? & \\
\hline & Geven & 1 & 10 & 72 & fir & 902 & bes & Wo & $\infty \times$ & 100 & en & Ge & 13 & 251 & 251 & kaction & \\
\hline Rev $66[0]$ & mentivetese & 1 & $x$ & 32 & 100 & 90 & hen & 500 & 50 & 190 & mi & 18. & 35 & 4.56 & 194 & Nessber. & Puscale \\
\hline & bove by tige" & I & 10 & 27 & 130 & G00 & bas. & 380 & 130 & 190 & 330 & aes. & 103 & 4.13 & 38 & ninove & \\
\hline
\end{tabular}

Best fitting model parameters for $a_{m i n}=1 \mu \mathrm{m}$ and $a_{\min }>1 \mu \mathrm{m}$ for each occultation. We present the results from both these particle size regimes because models with $a_{\text {min }}=1 \mu \mathrm{m}$ introduce a degeneracy in the results since such small particles diffract much of the light outside of the UVIS FOV. The results can fit the bowl-shaped occultation event well, but do not produce the clear diffraction "ears" observed outside of the F ring edges (see text for more details). Thus, while the observations that are best-fit with these particles may be indicative of a population of such small particles, we present the best-fit model results from both size regimes to look for a more likely trend between the effective particle size, equivalent depth, and the presence of the diffraction signature. The * indicates the occultation was best fit by a model particle size distribution with $a_{\min }>1$. 


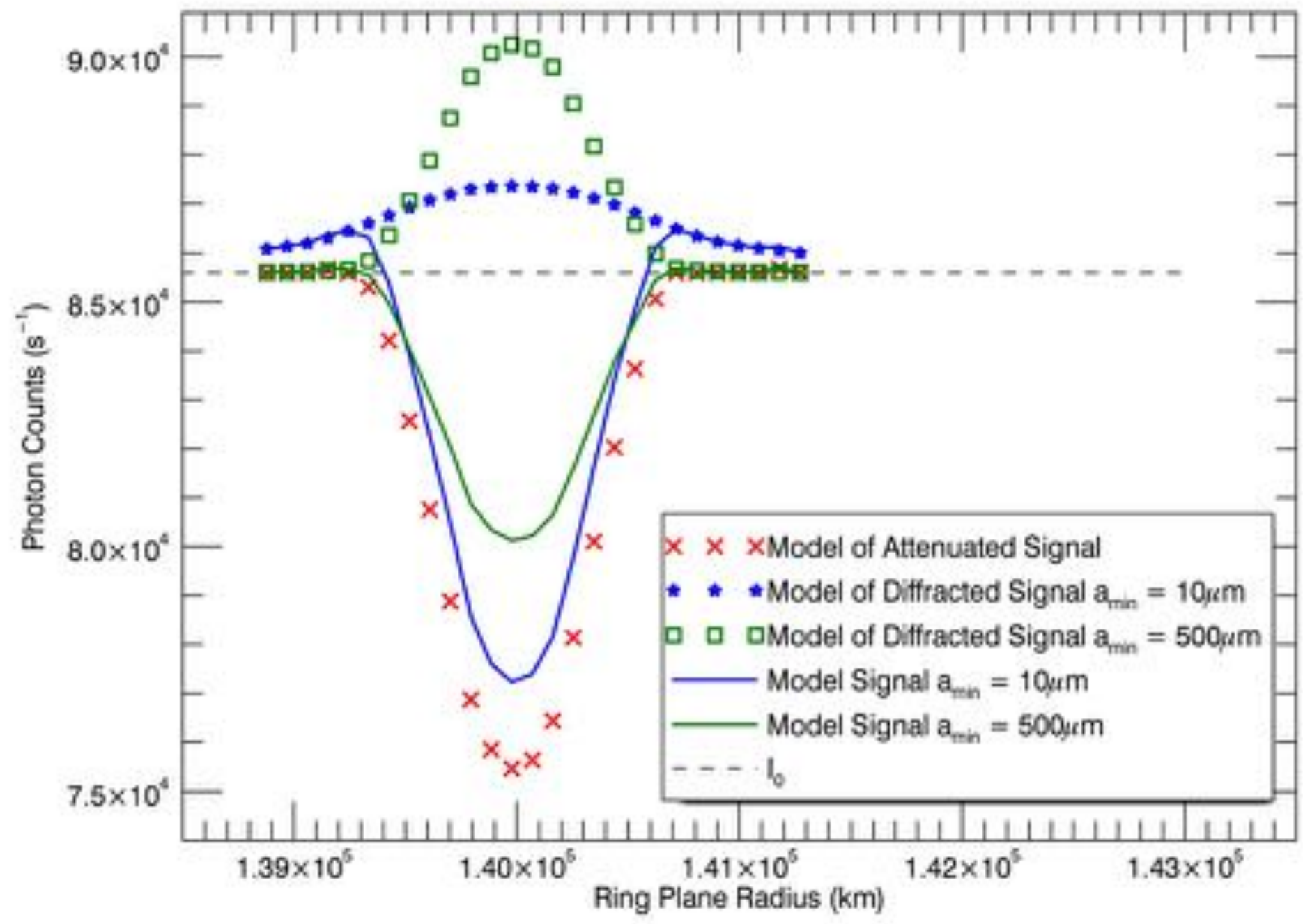




\begin{tabular}{|c|c|c|c|c|c|c|c|c|c|c|c|c|c|c|c|c|c|}
\hline \multirow[t]{2}{*}{ Oesulater } & \multirow[t]{2}{*}{ Anesergien } & \multicolumn{2}{|c|}{$\begin{array}{l}\text { Minimum } \\
\text { sartide dite } \\
\text { indereal }\end{array}$} & \multicolumn{2}{|c|}{ a } & \multicolumn{2}{|c|}{ thes } & \multicolumn{2}{|c|}{$\begin{array}{l}\text { Kinc Widh } \\
\text { Dinst }\end{array}$} & \multicolumn{2}{|c|}{$\begin{array}{l}\text { Ethective Particla } \\
\text { Sise [micosin] }\end{array}$} & \multicolumn{2}{|c|}{ 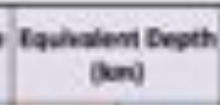 } & \multicolumn{2}{|c|}{$x_{v}^{2}$} & \multirow{2}{*}{$\begin{array}{l}\text { Betraction } \\
\text { Syeutive }\end{array}$} & \multirow{2}{*}{$\begin{array}{l}\text { Collivion } \\
\text { Stpuature }\end{array}$} \\
\hline & & Ium & $.1 \mathrm{~mm}$ & $10 \mathrm{~m}$ & $.31 \mathrm{re}$ & $1 \mathrm{gm}^{2}$ & bien & $1 \mathrm{y}^{\mathrm{n}}$ & nigh & $1 \mathrm{~m}$ & n $1 \mathrm{am}$ & $1 y=$ & pin & $10 n$ & niph & & \\
\hline \multirow{3}{*}{ Rer $43(0)$} & fuenine & 1 & $\sqrt{9}$ & 92 & 20 & 900 & 922 & 150 & 900 & 150 & 422 & T25 & 32 & 1572 & 185. & $7 \mathrm{n}$ & \multirow{3}{*}{$\mathrm{Fea}$} \\
\hline & merer Ning Edge & $t$ & 100 & 40 & 12 & ens & as? & inc & $\%$ & 10 & end & $\mathrm{j}=$ & is & $13 \mathrm{rr}$ & its & Wo & \\
\hline & over Rin fily" & i & 25 & 20 & 13 & aso & a.43 & 160 & 150 & 190 & 220 & 245 & 265 & 32 & 6.45 & $x_{n}$ & \\
\hline \multirow{3}{*}{ Rev 900} & Solvier & i & 25 & 13 & 15 & $95 t$ & aas & 160 & Jxa & 180 & $1 \%$ & IEs & 25 & tes & 28 & 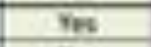 & \multirow{3}{*}{ sen } \\
\hline & nener kine fises. & 1 & 25 & 92 & 35 & 900 & $2 a 2$ & JSe & DS & 180 & 19 & Is & 25 & $x 4$ & 244 & $m_{n}$ & \\
\hline & aver bin the" & t. & 23 & 13 & 12 & les & 691 & 100 & 729 & 100 & 198 & is & 7.2. & 3.17 & 28 & wh & \\
\hline \multirow{3}{*}{ Berss $[t]$} & ruebive & 1 & 75 & 18 & 27 & 0,00 & a.95 & 900 & 500 & 200 & 20 & 15 & 125 & 430 & 67 & Tes & \multirow{3}{*}{ Sen } \\
\hline & inner Ning tege & 1 & 8 & 10 & 10 & $6 \infty 9$ & a.95 & 50 & sen & 10 & (2)3 & 116 & 207 & 43 & $2 n$ & 43 & \\
\hline & aver bivg fide" & 1 & 25 & 30 & 13 & 9.51 & a.s. & 30 & $\cos$ & 10 & 220 & 15 & is & 4.5 & sis & $7 e s$ & \\
\hline \multirow{3}{*}{ Rer 62 (5) } & fol $\operatorname{kins}^{2}$ & t & 10 & 32 & 27 & 90 & 6.73 & 300 & 82 & 180 & 222 & 118 & 174 & 262 & $28 \%$ & $7 n$ & \multirow{3}{*}{$\mathrm{Ev}$} \\
\hline & inner Ning Colye" & $i$ & 10 & 27 & 12 & $g=n$ & $a n$ & Jue & ma & ise & 203 & 135 & ins & 15 & 24 & 45 & \\
\hline & goen king fide" & t & $8 x$ & as & 33 & 90 & a.sas & ins & $\mathrm{Cos}$ & ins & 169 & 156 & 207 & IINA & gas & $m$ & \\
\hline \multirow{3}{*}{ Aev S9 (I) } & forenes & 1 & 10 & 37 & 27 & 902 & 693 & 700 & 50 & 10 & 531 & in & 184 & Den & 35 & W2 & \multirow{3}{*}{ Ne } \\
\hline & weat Hige tige" & 1 & 100 & 35 & 27 & 900 & b.253 & 190 & 500 & 100 & 539 & 183 & 123 & 0.72 & 27 & N) & \\
\hline & 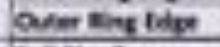 & 1 & 300 & 49 & 40 & 200 & 0.135 & 300 & 900 & 10 & 323 & if & 175 & 1 & 1.25 & St & \\
\hline \multirow{3}{*}{ Aev $62(0)$} & Fof Eivr? & 1 & 25 & 22 & 27 & 980 & 922 & 560 & 54 & 160 & 60 & 116 & $\longdiv { 1 1 6 }$ & 16 & ES & 45 & \multirow{3}{*}{ No } \\
\hline & Nerer Ming tege" & it & 3 & 22 & 27 & 200 & $6 \geq 2$ & 38 & 50 & 150 & 60 & 11.4 & its & ort & $25 ?$ & 40 & \\
\hline & aver kin fdys & $i$ & $x$ & 46 & 23 & e.pa & 2.23 & 210 & 200 & 10 & 240 & 35 & 13 & 231 & 252 & Wo & \\
\hline & Soeking & 1 & $1 x$ & 32 & 20 & s.98 & 9.73 & 100 & 60 & 10 & eis & 15 & B) & 70 & 255 & Roubbe & \\
\hline Aev 55 (1) & nner Ring Rolye" & $i$ & 5 & is & 15 & 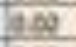 & as? & 40 & 60 & $\mathrm{mos}$ & 260 & is & to & 146 & 34 & Nowble & Oid collivien \\
\hline & oporkinget & 1 & $x$ & 32 & is & $\operatorname{leg}$ & has & $\infty$ & 100 & 140 & 132 & is & is & 2ss. & gix & S? & \\
\hline & forkest & 1 & 25 & 27 & 23 & 002 & 922 & 980 & 580 & 100 & 400 & 116 & 114 & 181 & 271 & 6ostive & \\
\hline ดev 65 (1) & mear bive tise & 1 & 25 & 10 & 27 & 900 & 292 & 790 & $\infty$ & 10 & 40 & 75 & 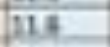 & 3.4 & $2 \pi$ & Nr & Me \\
\hline & berentives" & 1 & 10 & 23 & 35 & $\lg 0$ & $6 n$ & 960 & 500 & 190 & 19 & 115 & 10 & 182 & 25 & notitese & \\
\hline & We kive & 1 & 25 & 15 & is & $a n$ & 22 & 100 & 900 & 100 & 9 & 16 & 8 & 2.85 & 18 & Menber & \\
\hline Ber 65 [t] & ineer Miss Chye" & 2 & 300 & 25 & 27 & 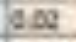 & a2x & 300 & 200 & 100 & 349 & 11.5 & 203 & 4.45 & 257 & 42 & Aoniple \\
\hline & Ouner Ring Idpa" & 1 & 5 & 20 & 40 & 9.00 & 2.22 & 300 & 900 & 170 & 30 & 11.6 & 10 & 1.H & 2.03 & Nonbie & \\
\hline & Foe Winct & $t$ & 25 & 34 & eb & $\overline{909}$ & a22 & 300 & 40 & 100 & 5 & 16 & $f 2$ & 13 & 57 & Rousbir & \\
\hline คrev 66 (1) & nent king filye" & 1 & 3 & 18 & 16 & $9: 98$ & bas & 30 & 192 & the & 82 & 118 & 25s & 307 & 193 & Rosibst & No \\
\hline & aver bes tilp & i & 25 & 13 & 40 & $a m$ & a.2. & 10 & $\operatorname{sen}$ & $m$ & 19 & $1=$ & te & 141 & [143 & s: & \\
\hline & astang & 1 & 10 & 22 & in & 908 & 2023 & 800 & 900 & 190 & 400 & in & 13 & 731 & 235 & hoction & \\
\hline Rer $66[t]$ & wert kire teke" & 1 & $x$ & 32 & 20 & 120 & 212 & 300 & $\omega$ & 190 & 23 & 12 & 3 & 4.46 & 19 & nessber. & Pusvale \\
\hline & 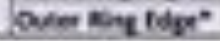 & it & 10 & 27 & 130 & 600 & lags & 350 & 180 & 190 & 930 & acs. & 105 & 413 & 31 & sinble & \\
\hline
\end{tabular}

